\title{
Detection of Cracks in Rotating Shafts by Using the Combination Resonances Approach and the Approximated Entropy Algorithm
}

\author{
Rodrigo Nicoletti $\mathbb{D}^{1},{ }^{1}$ Aldemir A. Cavalini Jr. ${ }^{(D)}{ }^{2}$ and Valder Steffen Jr. ${ }^{2}$ \\ ${ }^{1}$ Department of Mechanical Engineering, São Carlos School of Engineering, University of São Paulo, Trabalhador São Carlense 400, \\ 13566-590 São Carlos, SP, Brazil \\ ${ }^{2}$ LMEst-Structural Mechanics Laboratory, School of Mechanical Engineering, Federal University of Uberlândia, \\ Av. João Naves de Ávila 2121, 38408-196 Uberlândia, MG, Brazil
}

Correspondence should be addressed to Aldemir A. Cavalini Jr; aacjunior@ufu.br

Received 4 January 2018; Revised 12 March 2018; Accepted 19 April 2018; Published 17 May 2018

Academic Editor: Ivo Caliò

Copyright (C) 2018 Rodrigo Nicoletti et al. This is an open access article distributed under the Creative Commons Attribution License, which permits unrestricted use, distribution, and reproduction in any medium, provided the original work is properly cited.

\begin{abstract}
Shaft crack detection is a very serious matter and machines suspected of having a crack must be treated carefully. The importance attributed to this problem is addressed due to the serious consequences when cracks are not early detected in rotating systems. Various crack detection techniques were proposed in the last years, in which the vibration based techniques have demonstrated being efficient. However, these techniques fail for the cases in which incipient cracks are concerned. Recently, a nonlinear approach to detect cracks in rotating shafts was presented. The idea is to excite the shaft by using a harmonic force to induce combination resonances in the system. If the combination resonances appear in the vibration responses of the rotating system, the presence of cracks is confirmed. However, this methodology demonstrated being effective in detecting only deep cracks. In this context, the uniqueness of this paper relies on the possibility of detecting incipient transverse cracks in rotating shafts by associating the combination resonances approach with the so-called Approximated Entropy algorithm (ApEn algorithm). ApEn is a statistical value used to quantify irregularities in data series. Patterns and correspondences between samples of the same series are searched to detect anomalies. Considering that the combination resonances change the pattern of the shaft vibration responses, the ApEn algorithm can be used to highlight the presence of such resonances and, consequently, the detection of incipient cracks. The proposed approach was numerically evaluated by considering a horizontal rotating machine. A preliminary experimental investigation is also presented. The results demonstrated the efficiency of the conveyed methodology.
\end{abstract}

\section{Introduction}

The detection of cracks in shafts of rotating machines has attracted the attention of several researchers around the world. Different techniques were proposed in the last years, showing that the vibration based approaches are efficient in this goal [1-6]. Commonly, the $1 \mathrm{X}, 2 \mathrm{X}$, and, in certain cases, 3X vibration components are monitored for crack detection purposes [2]. However, various malfunctions in rotors may lead to similar symptoms. For instance, shaft angular misalignment can also be detected by monitoring $1 \mathrm{X}$, $2 \mathrm{X}$, and $3 \mathrm{X}$ vibration components [1]. Additionally, this signal based approach is able to detect cracks that have already propagated significantly across the shaft cross section. Therefore, sophisticated methods capable of identifying incipient cracks are of interest in the context of rotating machines.

Seibold and Weinert [7] identified the severity of cracks in rotors based on vibration measurements. In this case, a time domain identification algorithm was used considering the so-called Extended Kalman Filter. The identification is performed by building various filters, each one tuned to the hypothesis of a different damage severity. Probabilities for the different hypotheses are calculated and, consequently, the crack depth can be identified. The procedure demonstrated 
being effective in both numerical and experimental applications.

Alternatively, Sekhar [8] used the continuous wavelet transform to detect cracks in a rotating machine supported by hydrodynamic bearings. Vibration responses of the system measured during run-down were used for this purpose. In this case, peaks of subharmonic frequencies can be detected by using the wavelet transform, which are attributed to the presence of cracks. The author showed that such peaks can not be detected by the Fast Fourier Transform due to the nonstationary nature of the measured vibration responses.

Sinha [9] showed experimentally that higher order spectra analyses (bispectrum and trispectrum) can be used to distinguish malfunctions usually found in rotating machines, namely, crack and misalignment. The identification of higher harmonics in the system vibration responses is a typical case of nonlinear behavior. Breathing cracks are known to generate $1 \mathrm{X}, 2 \mathrm{X}$, and higher vibration components. Misalignment can also present such characteristics. However, although similar, the effects were separated by using higher order spectra analyses.

Kulesza and Sawicki [10] proposed a crack identification approach based on state observers. The rotor model was augmented by adding an auxiliary system of a single degree of freedom. As in [7], various state observers were designed for both the existence of the crack and its location. The resulting state variables were considered as crack indicators. Numerical simulations demonstrated the efficiency of the proposed methodology to detect and locate the crack in the shaft.

Mani et al. [11] were some of the precursors of an innovative crack detection technique based on the nonlinear effect induced by combination of resonances in rotating shafts. In their work, a Jeffcott rotor supported by rolling bearings was used to assess the potentiality of this technique. A breathing crack was detected by applying a specific diagnostic force to the system by using an electromagnetic actuator. According to them, if the diagnostic force is chosen properly, the presence of the crack creates spectral responses with peaks at frequencies that are combinations of the rotor speed, its critical speed, and the frequency of the diagnostic force. In this case, the Multiple Scales Method was used to determine the necessary conditions to induce combination resonances in the vibration responses of the system. The satisfactory results obtained by using the combination resonances approach for crack detection purposes have guided other works [1214].

Sampaio and Nicoletti [15] applied the Approximated Entropy (ApEn) algorithm to detect cracks in a rotating shaft. The ApEn is a statistical value used to quantify irregularities in data series. Patterns and correspondences between samples of the same series are searched to detect anomalies. Numerical simulations demonstrated the feasibility of the proposed methodology, detecting cracks down to $5 \%$ crack depths. According to the authors, the ApEn algorithm could differentiate the occurrence of crack only, misalignment only, and both found simultaneously in the system. However, satisfactory results were obtained only by considering the rotor operating under run-up conditions (nonstationary tests).
In this context, this paper proposes to associate the combination resonances approach with the ApEn algorithm to detect cracks in shafts of rotating machines. Following the methodology presented by Cavalini Jr. et al. [14], the vibration responses of the rotor operating at constant speed are measured for different diagnostic forces. Considering that the combination resonances change the pattern of the shaft vibration responses, the ApEn algorithm is used to highlight the existence of such resonances and, consequently, the detection of incipient cracks. In the present contribution, numerical simulations and a preliminary experimental test were performed to demonstrate the efficiency of the proposed approach. A horizontal rotor system composed of a flexible shaft, two discs, and two ball bearings was used in the numerical simulations and in the experimental application conveyed. It becomes clear from the results that the association of the ApEn algorithm with the combination resonances approach facilitates the identification of cracks in the rotating system.

\section{Combination Resonances}

As mentioned, the combination resonances approach for detecting cracks in shafts was previously presented by Mani et al. [11] and it is now being improved by other researchers [12-14]. The idea is to excite the rotating system (or the structure where the rotating system is mounted on) with a harmonic force of frequency $\Omega_{d}$, and such force will induce combination resonances in the rotor. By detecting these combinations in the vibration responses of the system, it is possible to identify a crack along the shaft. According to Mani et al. [11], if there is a crack in the shaft such combinations will occur for

$$
\omega_{\Omega}= \pm \Omega_{d}+n \Omega,
$$

where $\omega_{\Omega}$ is a critical speed of the rotor (backward or forward whirls), $\Omega$ is the rotating speed, and $n$ is an integer number $( \pm 1, \pm 2, \ldots)$. Hence, the frequency $\Omega_{d}$ of the diagnostic force is defined as a multiple of the rotating speed plus the critical speed of choice:

$$
\Omega_{d}=\omega_{\Omega} \pm n \Omega .
$$

2.1. The Rotating System. The combination resonances approach is applied to a mathematical model of a cracked rotating system. The rotating system is composed of two roller bearings supporting a flexible steel shaft with $860 \mathrm{~mm}$ length and $17 \mathrm{~mm}$ diameter. The flexible shaft contains two steel disks of $150 \mathrm{~mm}$ diameter and $20 \mathrm{~mm}$ thickness (see Figure 1(a)). A finite element model (FE model) of the rotating system (Figure 1(b)) based on Timoshenko's beam elements leads to the following equations of motion:

$$
\mathbf{M} \ddot{\mathbf{q}}+\left[\mathbf{D}+\Omega \mathbf{D}_{g}\right] \dot{\mathbf{q}}+\mathbf{K q}=\mathbf{f}_{w}+\mathbf{f}_{u}+\mathbf{f}_{e},
$$

where $\mathbf{q}$ is the vector of generalized displacements, $\mathbf{M}$ is the inertia matrix, $\mathbf{D}$ is the damping matrix, $\mathbf{D}_{g}$ is the gyroscopic matrix, $\mathbf{K}$ is the stiffness matrix, $\mathbf{f}_{w}$ is the gravity force vector (weight), $\mathbf{f}_{u}$ is the unbalance force vector, and $\mathbf{f}_{e}$ is the diagnostic force vector (associated with $\Omega_{d}$ ). 


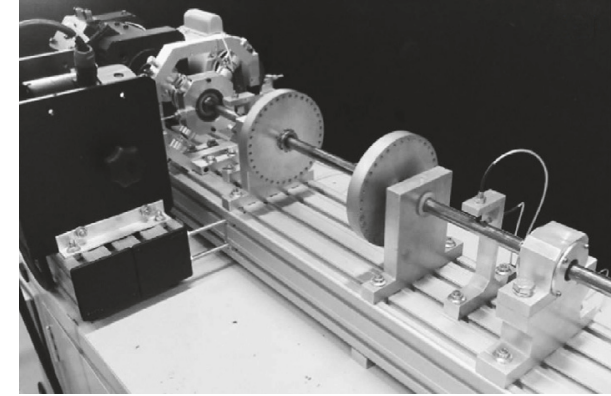

(a)

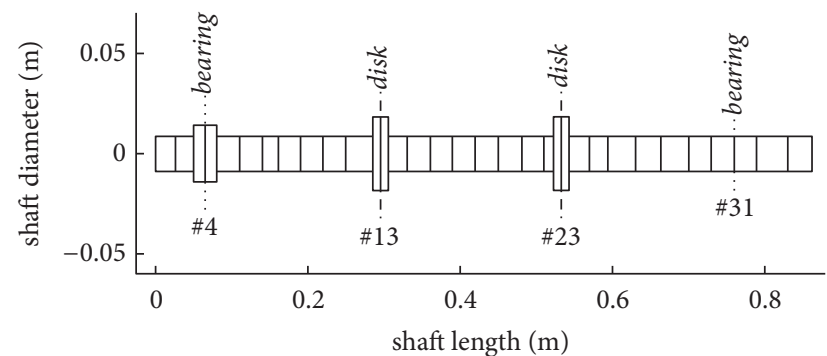

(b)

FIGURE 1: Rotating system: (a) test rig (real system); (b) FE model [14].
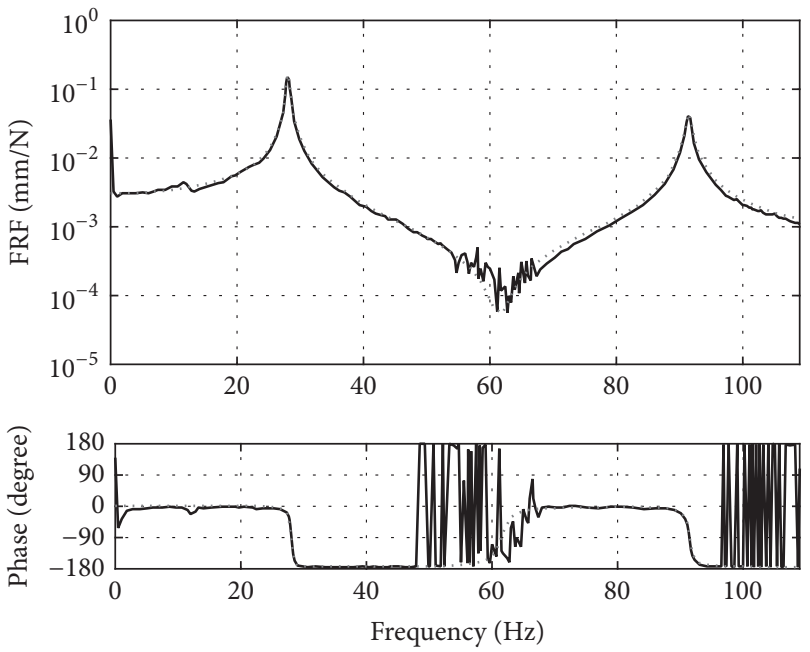

experiment

model

FIGURE 2: FRF obtained at node \#8 of the FE model with excitation on node \#13 and the corresponding experimental result [14].

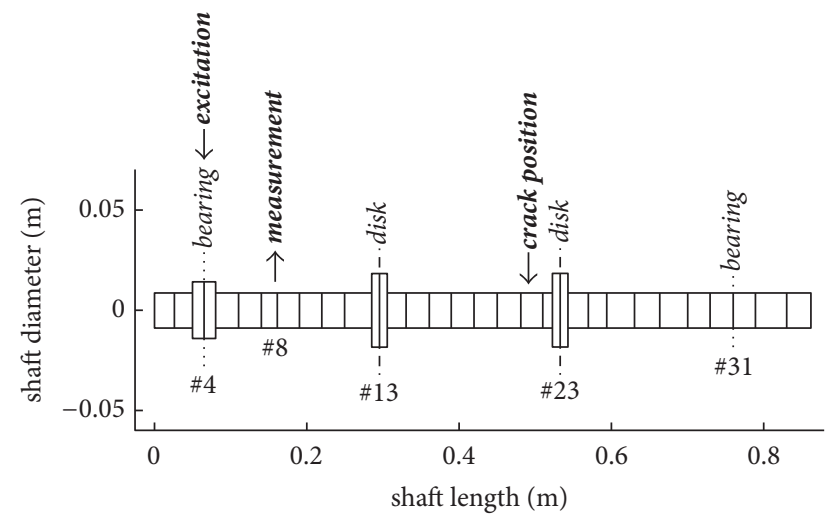

FIGURE 3: Location of excitation and measurement points for the FE model.

The rotor FE model (without crack) was correlated to experimental results by using the Differential Evolution optimization technique. The updated model resulted in the frequency response function (FRF) shown in Figure 2 for the rotor at rest (FRF of the rotating system obtained at node $\# 8$ as excited at node \#13-disk location). The first natural frequency of the system is $28.7 \mathrm{~Hz}$. Details of the model updating procedure are presented in Cavalini Jr. et al. [14].

The crack is modeled according to the theory presented by Mayes and Davies [16] for a breathing behavior. In this case, the stiffness matrix of the shaft FE model that contains the crack is composed of the stiffness matrix without crack associated with the flexibility matrix of the breathing crack, which is time variant according to

$$
\mathbf{K}(\Omega t)=\mathbf{K}+\mathbf{K}_{\mathrm{CR}}(\Omega t),
$$

where $\mathbf{K}_{\mathrm{CR}}(\Omega t)$ is a function of the crack depth and the angular position $\Omega t$ of the shaft (a thorough deduction of this matrix is presented in Cavalini Jr. et al. [14]).

Hence, the equation of motion of the shaft is now a matrix differential equation with time variant coefficient when the breathing crack is

$$
\mathbf{M} \ddot{\mathbf{q}}+\left[\mathbf{D}+\Omega \mathbf{D}_{g}\right] \dot{\mathbf{q}}+\mathbf{K}(\Omega t) \mathbf{q}=\mathbf{f}_{w}+\mathbf{f}_{u}+\mathbf{f}_{e} .
$$

2.2. Application of the Combination Resonances. To apply the combination resonances approach to crack detection, the frequency $\Omega_{d}$ of the diagnostic force is calculated from (2). Considering that the first forward critical speed of the rotating machine is $\omega_{\Omega}=28.7 \mathrm{~Hz}$ and choosing $n=+1$, the diagnostic frequency will be $48.7 \mathrm{~Hz}$.

First, the rotating system for the pristine condition rotates at $1200 \mathrm{rpm}(20 \mathrm{~Hz})$. By exciting the shaft at node \#4 (bearing location) with a diagnostic force of $25 \mathrm{~N}$ amplitude and $48.7 \mathrm{~Hz}$ frequency, the vibration response at node \#8 is calculated (see Figure 3; excitation and vibration responses obtained along the horizontal direction).

Figure 4 presents the Fast Fourier Transform (FFT) of the vibration responses obtained at node \#8 of the healthy FE model (pristine condition) when subjected to the diagnostic harmonic force. The results clearly show two main components in the spectrum, one at $20 \mathrm{~Hz}$ related to the rotating speed and another at $48.7 \mathrm{~Hz}$ related to the diagnostic excitation. Considering that the shaft has no crack, the spectrum shown in Figure 4 is considered as a reference for the health monitoring of the machine.

Four different crack depths of $1 \%, 3 \%, 5 \%$, and $10 \%$ shaft diameters were included in the FE model. The diagnostic 


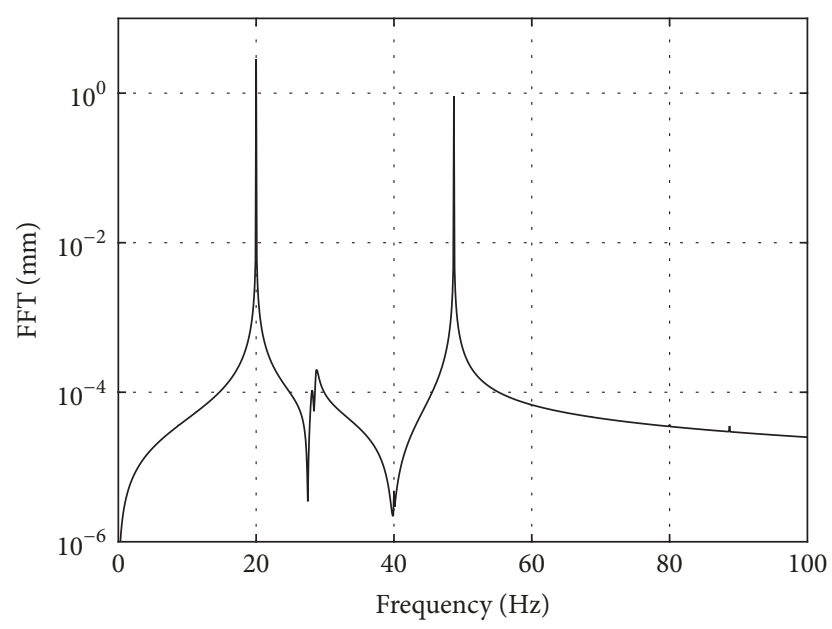

FIgURE 4: FFT of the system vibration responses at node \#8 as excited by the diagnostic frequency of $48.7 \mathrm{~Hz}$ at node \#4 (rotating speed of $1200 \mathrm{rpm}$ ).

excitation was repeated considering the same operating conditions as those adopted for the pristine condition. The crack is imposed to the element between nodes \#20 and \#21, as shown in Figure 3. Figure 5 shows that the bigger the crack depth is, the more the combination resonances occurs. As the crack depth increases, the peaks at the combination resonances become more evident and, consequently, it becomes easier to identify the crack. In this case (Figure 6), not only are the harmonics of the rotating speed identified $(1 \times, 2 \times$, $3 \times$, and $4 \times$ vibration components), but also the combination resonances at $\Omega_{d}+n \Omega$ (for $n=-2,-1,+1,+2$ ) and at $-\Omega_{d}+n \Omega$ (for $n=3,4$ ) are obtained.

However, it is much harder to identify the crack in the presence of noise. Figure 7 shows the same results when the FFTs have a signal-to-noise ratio (SNR) of 1000, being

$$
\mathrm{SNR}=20 \log _{10}\left(\frac{\mathrm{RMS}_{\mathrm{FFT}}}{\mathrm{RMS}_{\text {noise }}}\right),
$$

where $\mathrm{RMS}_{\mathrm{FFT}}$ is the root mean square value of the frequency domain rotor vibration responses and $\mathrm{RMS}_{\text {noise }}$ is the root mean square value of the noise signal.

The results show that the peaks at the combination resonances merge into the noisy spectrum, making it difficult to detect the crack. This situation is even more complicated for incipient cracks (crack depths of 1\% and 3\%; see Figures $7(\mathrm{a})$ and $7(\mathrm{~b}))$. For this reason, the combination of the present nonlinear approach with the ApEn algorithm can significantly improve the results and contribute to the better detection of incipient cracks in the shaft.

\section{The Approximated Entropy (ApEn) Algorithm}

The Approximated Entropy (ApEn) is a statistical value used to quantify the irregularity of data series [17]. According to Sampaio and Nicoletti [15], the ApEn algorithm can be used to search for patterns inside the series and to search for the correspondence of a data sample to another data sample of the same series. Therefore, anomalies can be detected in the series. Considering that the combination resonances approach changes the pattern of the shaft vibration responses as represented by their FFTs, the ApEn algorithm can be used to highlight the presence of such resonances, to help the early detection of the crack in the system, and to overcome the problems found in noisy signals.

In the ApEn algorithm, a data series of $N$ points is divided into blocks of $p$ data points. The first block of $p$ data points is considered correspondent to another sample of $p$ data points of the same data series if the maximum distance between the samples (defined by a norm) lies within a tolerance value $r$. Such procedure is repeated for samples of $p+1$ data points and the conditional probability of this new sample to be still correspondent to the later ones is calculated. At the end, the ApEn value is determined from the conditional probabilities found for $p$ data points and for $p+1$ data points. It will correspond to the ApEn value of the data series of $N$ points. Therefore, the ApEn value can be calculated at each set of $N$ points of a given signal. A complete description of the algorithm is presented in Sampaio and Nicoletti [15].

3.1. Application of the ApEn Algorithm. The first step to use the ApEn algorithm is the definition of the size $N$ of the data series (frequency at which the ApEn values are calculated). According to Sampaio and Nicoletti [15], the appropriate choice of the series dimension used to calculate the ApEn value (parameter $N$ ) is a compromise solution between filtering out nonuseful information and capturing the useful information in the signal. If $N$ is too large, the ApEn algorithm will average all information in the data series and the anomalies will not be highlighted by the ApEn value. If $N$ is too small, the ApEn algorithm will show every information in the data series and the anomalies will be lost amidst other information contained in the signal.

Figures 5 and 7 present FFTs with $0.1 \mathrm{~Hz}$ frequency resolution (10 data points per $\mathrm{Hz}$ ) and the results are shown up to $100 \mathrm{~Hz}$ (1000 data points in total). If the ApEn algorithm is applied to the whole series of 1000 points, a single ApEn value will be obtained, which does not give much insight into the FFTs' anomalies. If the ApEn algorithm is calculated at every 2 or 3 points of the FFT (at every 0.2 or $0.3 \mathrm{~Hz}$ ), it will give too much information and the anomalies will be hardly identifiable. In the present case, $N=19$ points is adopted to have an ApEn value at, approximately, every $2 \mathrm{~Hz}$. The use of odd and prime numbers for parameter $N$ helps to keep the harmonics and the combination resonances in the middle of the $N$ points series. When the parameter $N$ is odd, the combination resonances (anomalies in the FFT) may occur exactly at the beginning or at the end of the data series and, consequently, the calculation of the ApEn value is jeopardized. Figure 8 presents the results obtained by applying the ApEn algorithm to the FFTs shown in Figures 4 and 5 (no noise in the signals). In this case, $N=19$ (frequency at which the ApEn values are calculated), $p=2$ (size of the blocks in the ApEn algorithm), and $r=10^{-5}$ (tolerance adopted in the ApEn algorithm). 


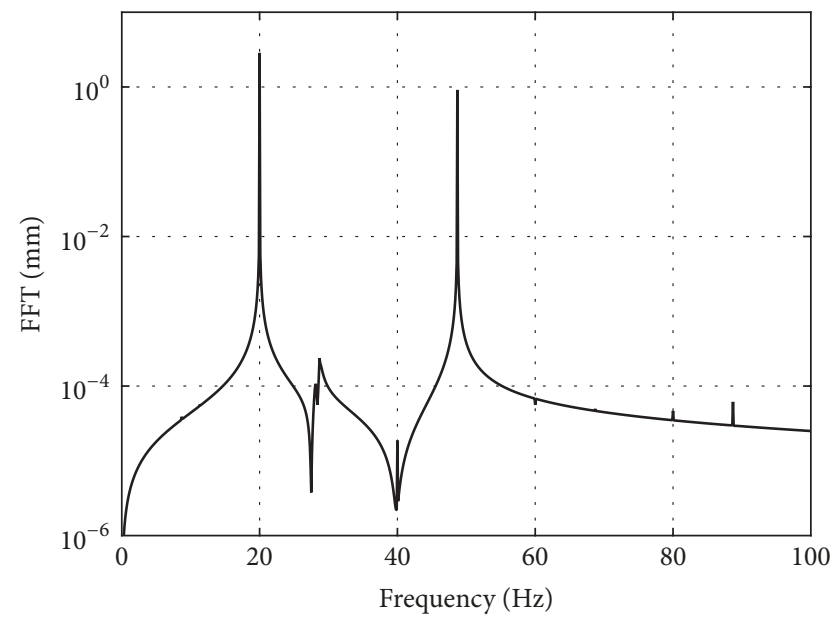

(a)

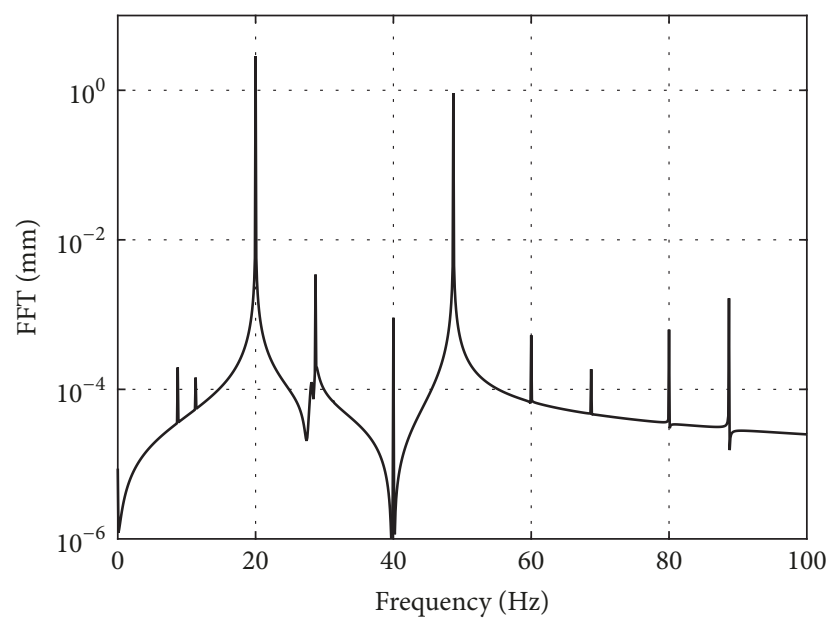

(c)

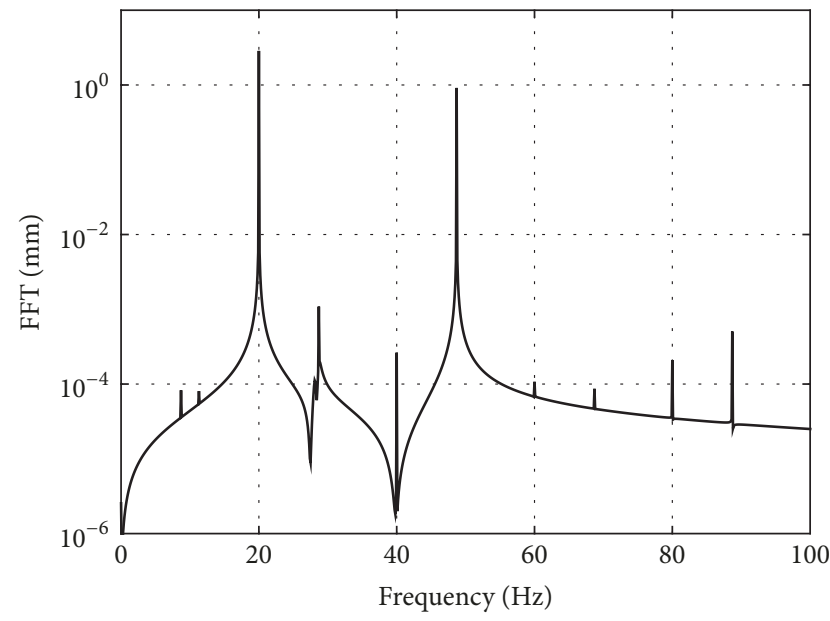

(b)

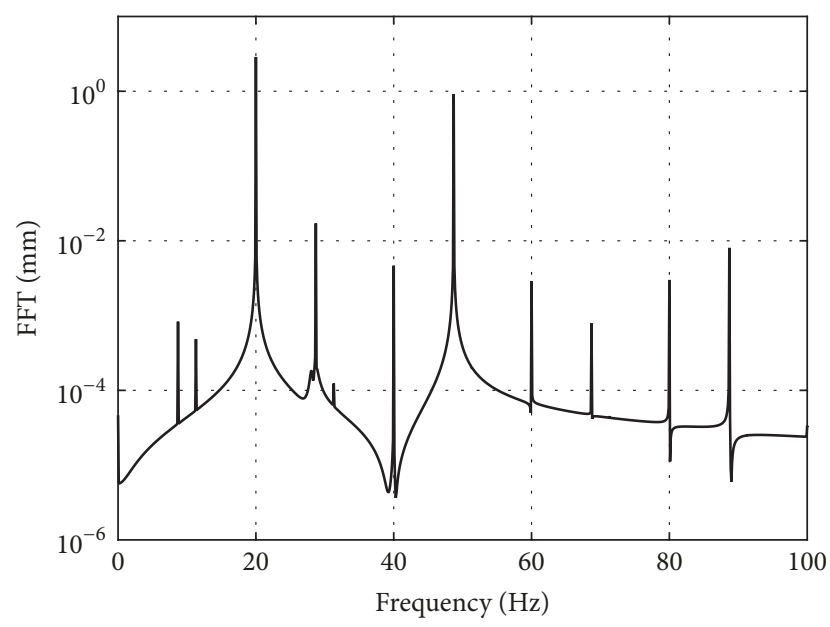

(d)

FIGURE 5: FFT of the system vibration responses at node \#8 when excited by the diagnostic frequency of $48.7 \mathrm{~Hz}$ at node \#4 (rotating speed of $1200 \mathrm{rpm}$ ): (a) $1 \%$ crack depth; (b) $3 \%$ crack depth; (c) $5 \%$ crack depth; (d) $10 \%$ crack depth.

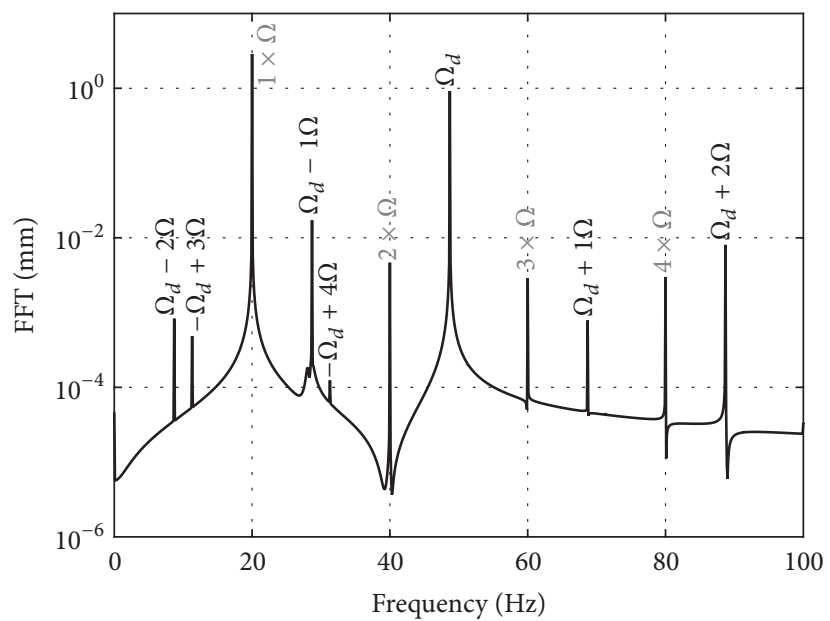

FIGURE 6: Identification of the combination resonances in the FFT of the system vibration responses with $10 \%$ crack depth. 


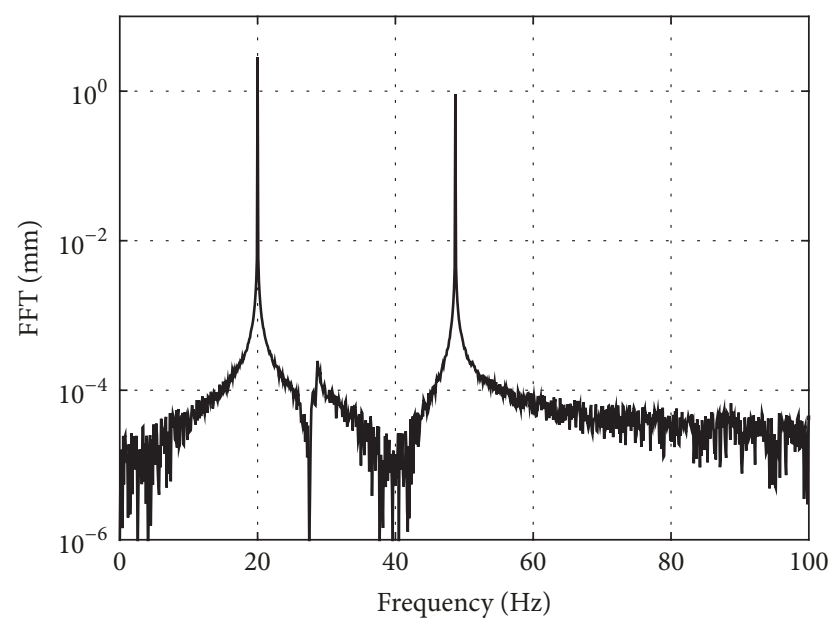

(a)

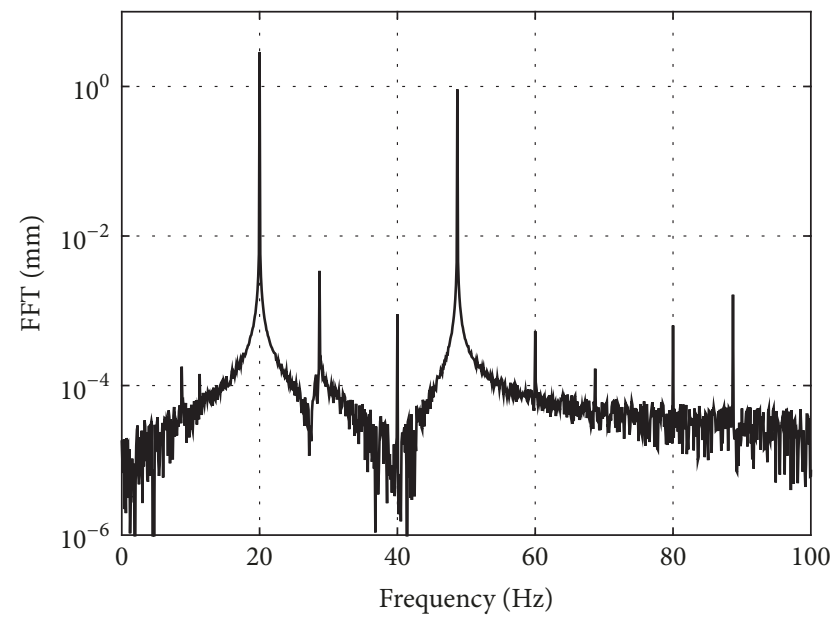

(c)

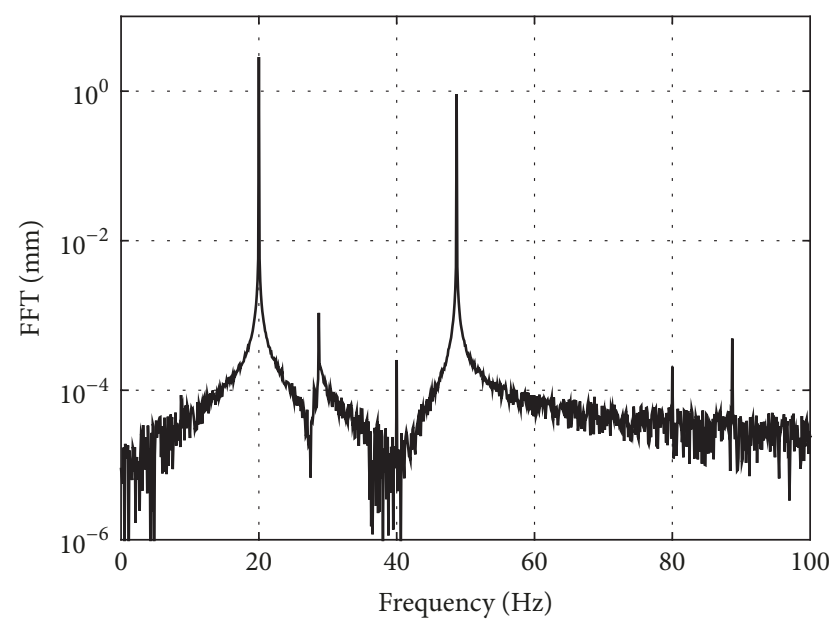

(b)

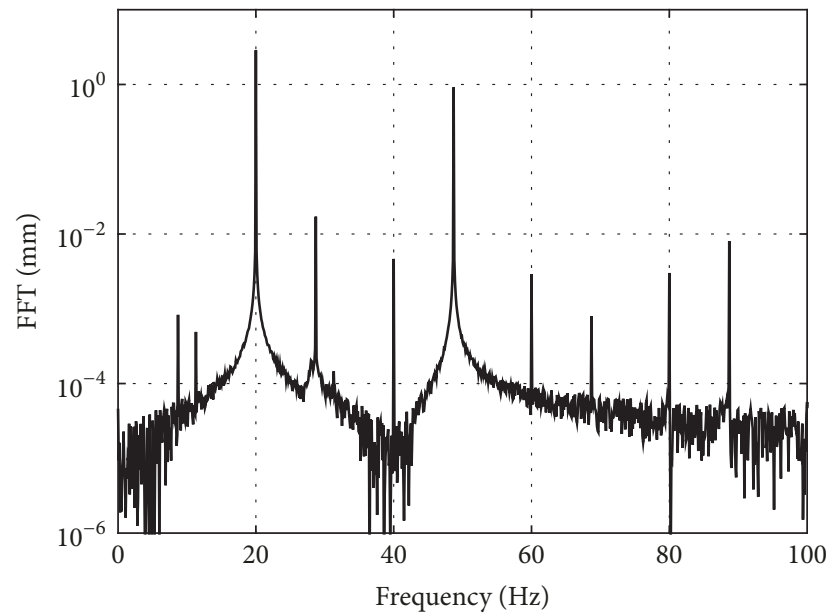

(d)

FIGURE 7: FFT of the system vibration responses at node \#8 as excited by the diagnostic frequency of $48.7 \mathrm{~Hz}$ at node \#4 (rotating speed of $1200 \mathrm{rpm}$ and SNR = 1000): (a) $1 \%$ crack depth; (b) 3\% crack depth; (c) $5 \%$ crack depth; (d) $10 \%$ crack depth.

Regarding the ApEn values of the FFT from the healthy shaft (black solid line in Figure 8), it can be observed that most of the information is concentrated between 10 and $60 \mathrm{~Hz}$. This result is expected, as the peaks in the FFT of Figure 4 are found in this frequency range and there are no anomalies elsewhere (no combination resonances or harmonics of the rotating speed). However, when there is a $1 \%$ crack depth (incipient crack; see Figure 8(a)) in the shaft, the combination resonances and the harmonics of the rotating speed appear. Consequently, more information appears in the FFTs beyond the frequency range of 10 and $60 \mathrm{~Hz}$. The ApEn value clearly indicates the presence of the $3 \times(60 \mathrm{~Hz})$ and $4 \times(80 \mathrm{~Hz})$ vibration components and the combination resonances $\Omega_{d}+2 \Omega(88.7 \mathrm{~Hz})$.

For the cases of $3 \%$ and $5 \%$ crack depths (Figures $8(\mathrm{~b}$ ) and $8(\mathrm{c})$ ), the ApEn values indicate the presence of vibration harmonics of the rotating speed and combination resonances at $\Omega_{d}-2 \Omega(8.7 \mathrm{~Hz}), \Omega_{d}+1 \Omega(68.7 \mathrm{~Hz})$, and $\Omega_{d}+2 \Omega(88.7 \mathrm{~Hz})$. In the case of a $10 \%$ crack depth (Figure $8(\mathrm{~d})$ ), the ApEn values indicate the presence of harmonics and combination resonances at $\Omega_{d}-2 \Omega(8.7 \mathrm{~Hz}), \Omega_{d}+1 \Omega(68.7 \mathrm{~Hz}), \Omega_{d}+$ $2 \Omega(88.7 \mathrm{~Hz})$, and $-\Omega_{d}+4 \Omega(31.3 \mathrm{~Hz})$. Hence, the presence of such peaks in the FFTs can be easily identified by using the ApEn algorithm. The ApEn values clearly highlight the anomalies in the FFTs.

3.2. Effect of Noise in the FFTs. Section 2.2 shows that it is more difficult to identify the existence of combination resonances and, consequently, the presence of cracks when the FFTs are noisy. The reason is that the resultant noisy FFTs (see Figure 7) hide the peaks at the combination resonances especially for incipient cracks ( $1 \%$ and $3 \%$ crack depths).

Figure 9 presents the ApEn values of the noisy FFTs shown in Figure 7, adopting the same parameters used in the previous analysis $\left(N=19, p=2\right.$, and $\left.r=10^{-5}\right)$. For these conditions, the ApEn algorithm does not distinguish the combination resonances from the noise peaks. The ApEn curve for the healthy system is very similar to the one corresponding to the cracked shaft. Thus, it is not possible to identify the existence of the combination resonances. 


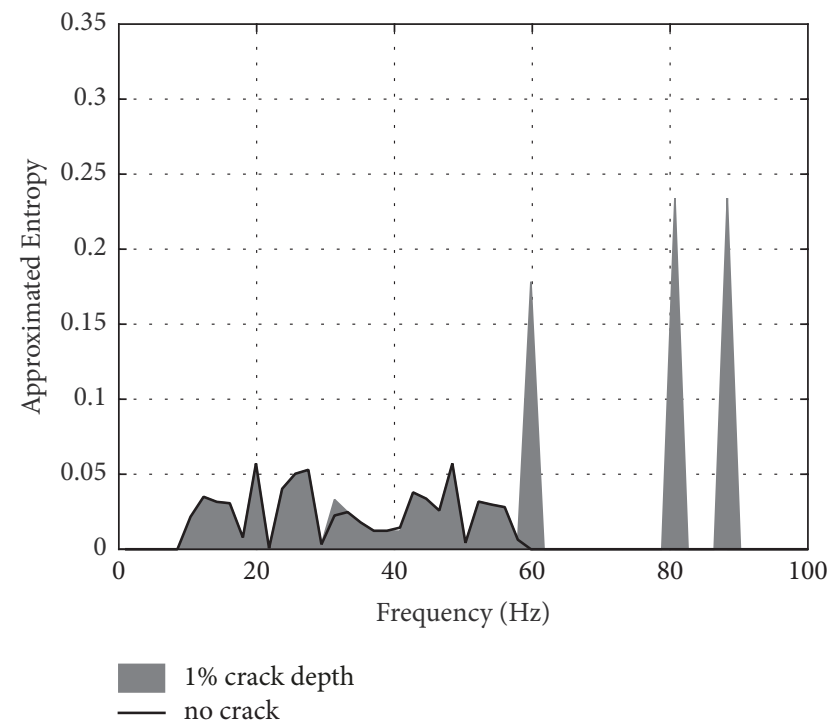

(a)

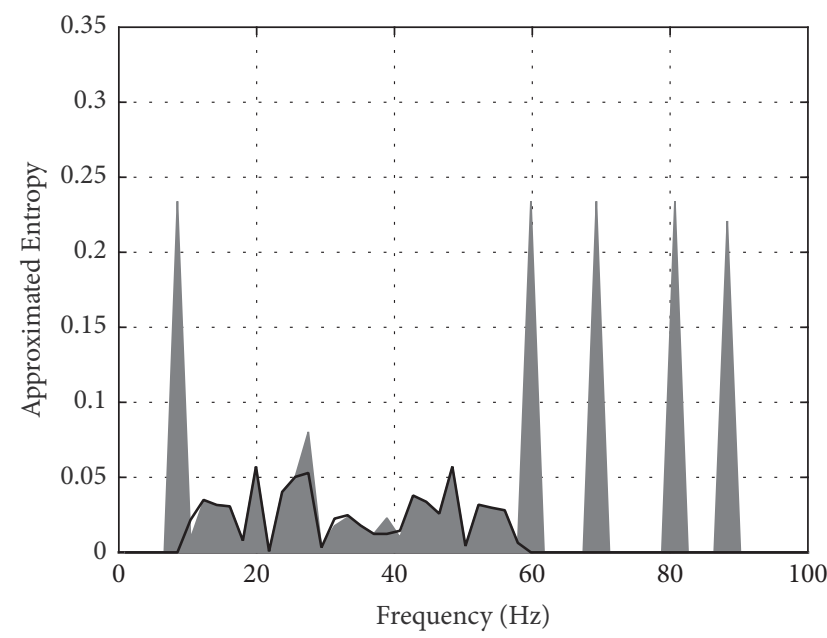

$5 \%$ crack depth no crack

(c)

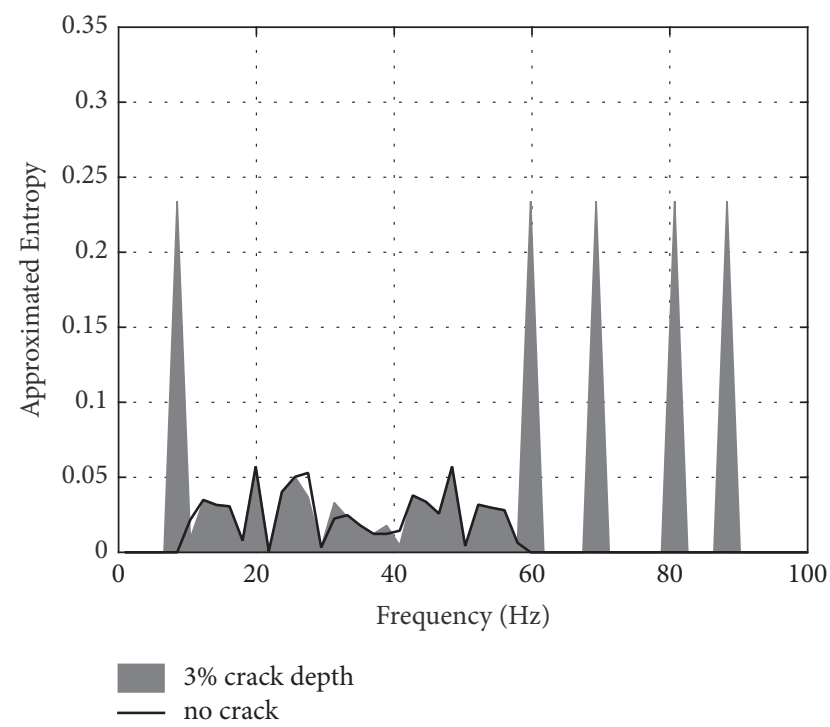

(b)

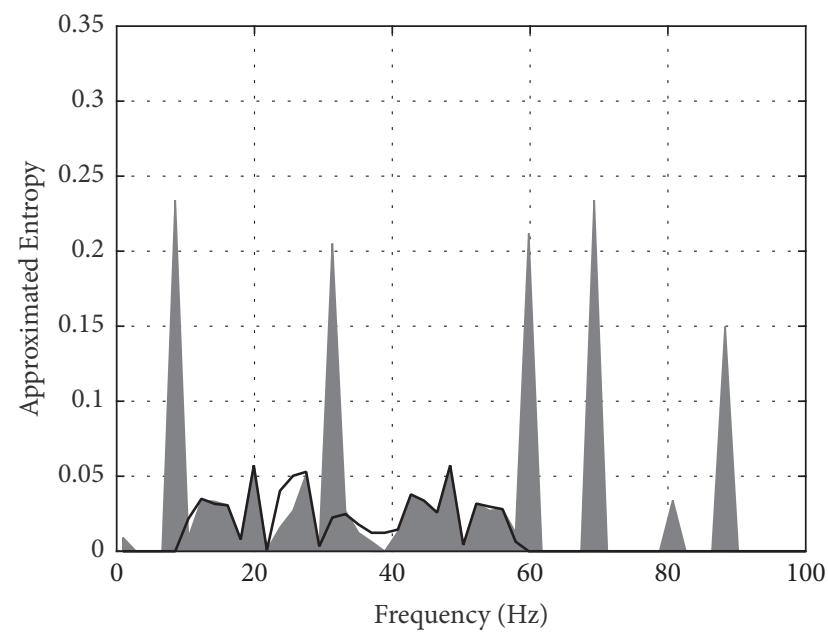

$10 \%$ crack depth no crack

(d)

Figure 8: Approximated Entropy of the FFTs with no noise. Comparison between the cases of healthy and cracked shaft: (a) $1 \%$ crack depth; (b) $3 \%$ crack depth; (c) $5 \%$ crack depth; (d) $10 \%$ crack depth.

However, by adjusting the tolerance of the ApEn algorithm (parameter $r$ ) to $5 \times 10^{-5}$, it becomes much clearer that there are peaks of combination resonances in the FFTs (see Figure 10). For the $1 \%$ crack depth (Figure 10(a)), a peak at $\Omega_{d}+2 \Omega(88.7 \mathrm{~Hz})$ can be observed. Regarding the $3 \%$, $5 \%$, and $10 \%$ crack depths (Figures $10(\mathrm{~b})-10(\mathrm{~d})$, resp.), there are combination resonances at $\Omega_{d}-2 \Omega(8.7 \mathrm{~Hz}), \Omega_{d}+1 \Omega$ $(68.7 \mathrm{~Hz}), \Omega_{d}+2 \Omega(88.7 \mathrm{~Hz})$, and $3 \times(60 \mathrm{~Hz})$ and $4 \times(80 \mathrm{~Hz})$ vibration components of the rotating speed. Consequently, it is possible to detect the presence of the crack in the system even for incipient cracks ( $1 \%$ and 3\% crack depths).

Hence, the parameter $r$ of the ApEn algorithm must be adjusted according to the level of noise in the FFTs. In fact, for different levels of SNR in the FFTs, the best results are obtained when adopting the values shown in Figure 11, which gives the following rule:

$$
r=\frac{0.05}{\mathrm{SNR}}
$$

and adopting $N=19$ and $p=2$, it is possible to obtain the ApEn results shown in Figure 12.

In Figure 12(a) (1\% crack depth), the ApEn algorithm highlights the combination resonances above an SNR of 1000 in the FFT. Below this level, it is not possible to observe the presence of the peaks and, consequently, the existence of the crack in the shaft. On the other hand, for crack depths of 3\% and above (Figures 12(b)-12(d)), it is possible to detect the presence of combination resonances above a 


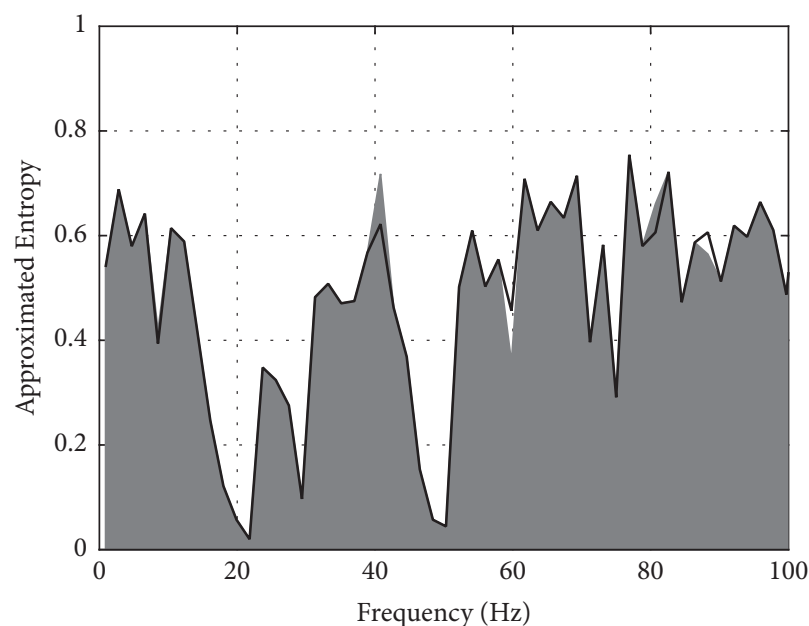

$1 \%$ crack depth no crack

(a)

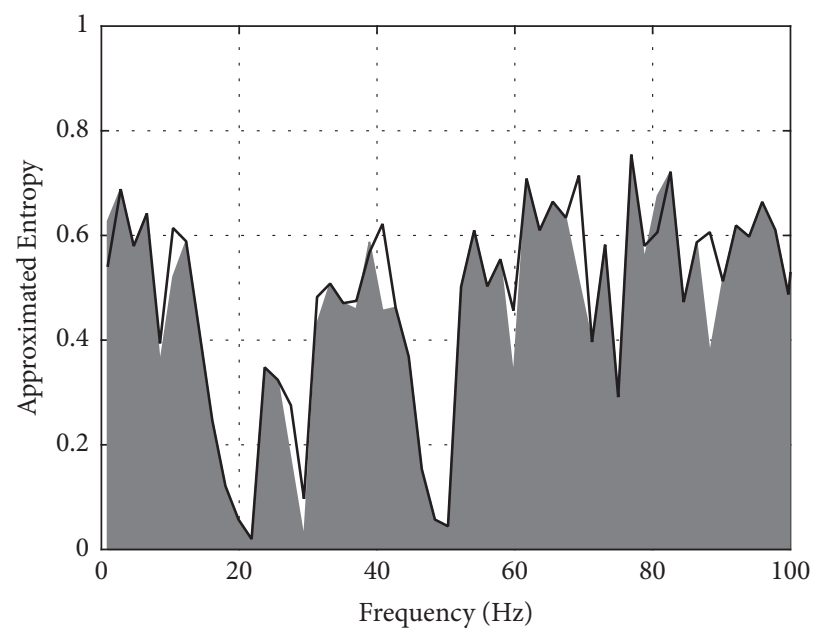

$5 \%$ crack depth no crack

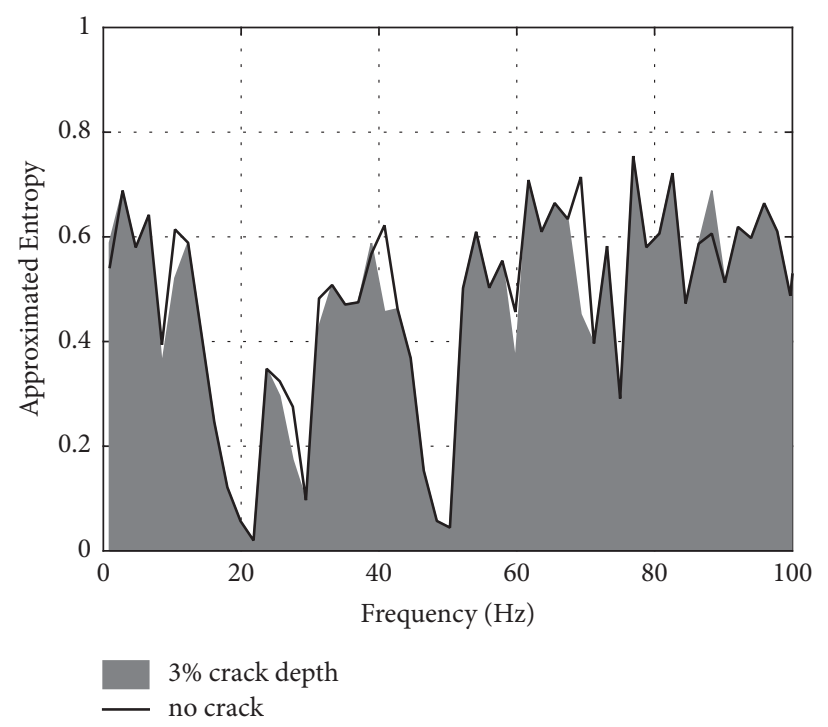

(b)

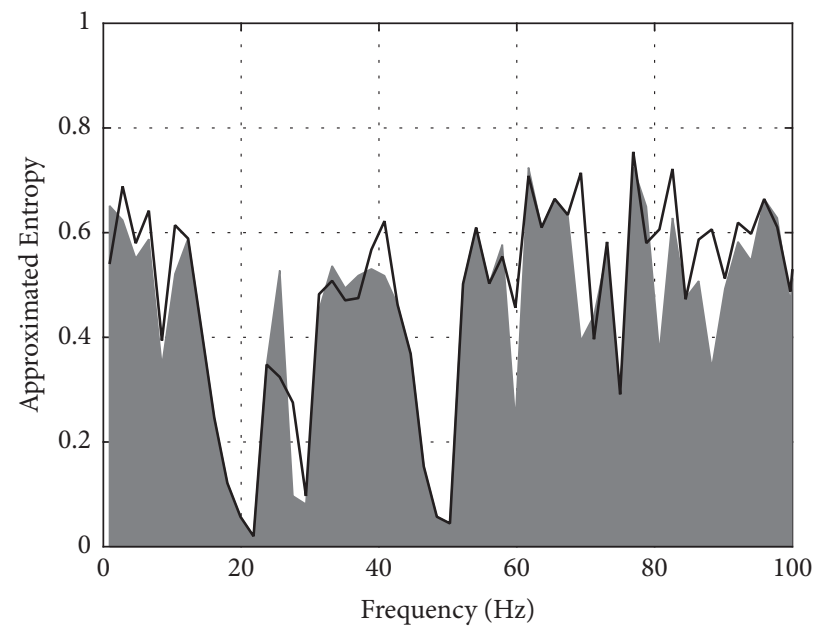

$10 \%$ crack depth no crack

(c)

(d)

Figure 9: Approximated Entropy of the FFTs with noise ( $\mathrm{SNR}=1000, r=10^{-5}$ ). Comparison between the cases of healthy and cracked shaft: (a) $1 \%$ crack depth; (b) $3 \%$ crack depth; (c) $5 \%$ crack depth; (d) $10 \%$ crack depth.

SNR of 100. Therefore, it is feasible to detect incipient cracks down to $3 \%$ crack depths from noisy FFTs by using the proposed nonlinear technique in combination with the ApEn algorithm.

It is clear in Figure 12 that as the SNR decreases the ApEn algorithm becomes less efficient on highlighting the presence of the combination resonances in the FFTs (see the number of peaks in Figure 12(b) as the SNR decreases). However, it is also important to note that an FFT with a SNR of 500 or below is very noisy and it would be hardly accepted in engineering practice.

3.3. Analysis of Repeatability. The results presented in Figure 12 were obtained by adding noise to the original FFT of the system. In this process, the noise is created from uniformly distributed random numbers, whose RMS amplitude is tuned according to the desired SNR. Hence, the results presented in Figure 12 were obtained from a single set of random numbers. To check for the robustness of the methodology, the process shall be repeated for different sets of random numbers.

Regarding the FFTs with SNR of 1000 (Figure 10), three combination resonances appeared as peaks in the ApEn curves, namely, $\Omega_{d}-2 \Omega(8.7 \mathrm{~Hz}), \Omega_{d}+1 \Omega(68.7 \mathrm{~Hz})$, and $\Omega_{d}+2 \Omega(88.7 \mathrm{~Hz})$. The amplitudes of these peaks are shown in Figure 13 as a function of the adopted sets of random numbers (runs). In this case, the process was repeated 300 times (300 different sets of random numbers were used to simulate noise in the FFTs).

For a crack depth of $1 \%$ (Figure 13(a)), there are no peaks at $8.7 \mathrm{~Hz}$ and $68.7 \mathrm{~Hz}$ (zero amplitude). In addition, 


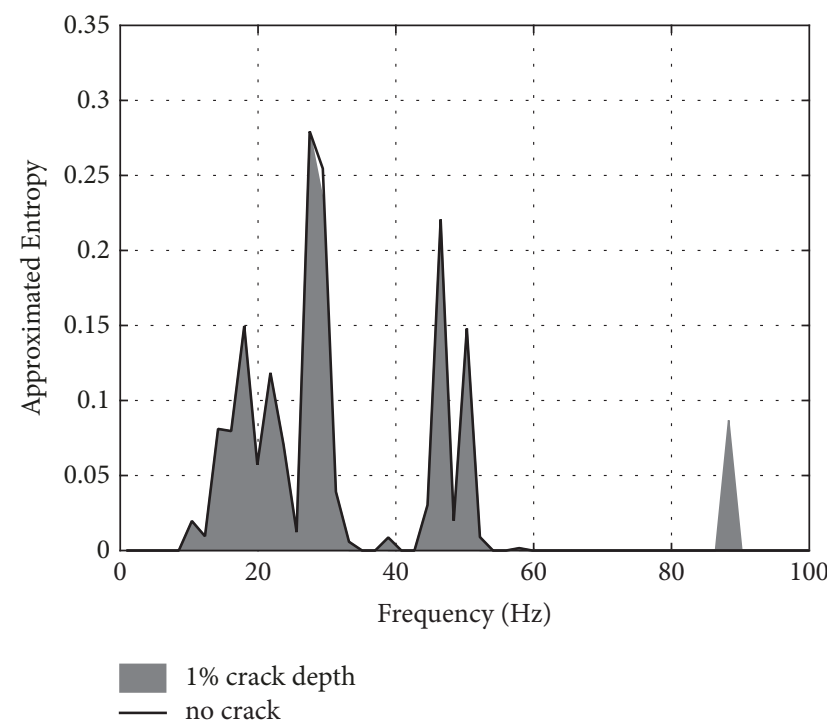

(a)

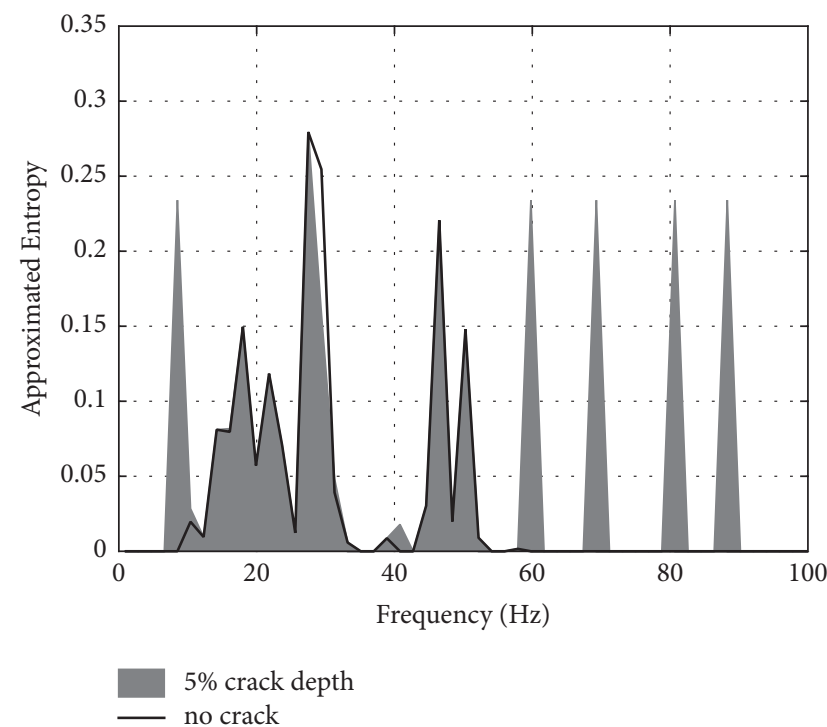

(c)

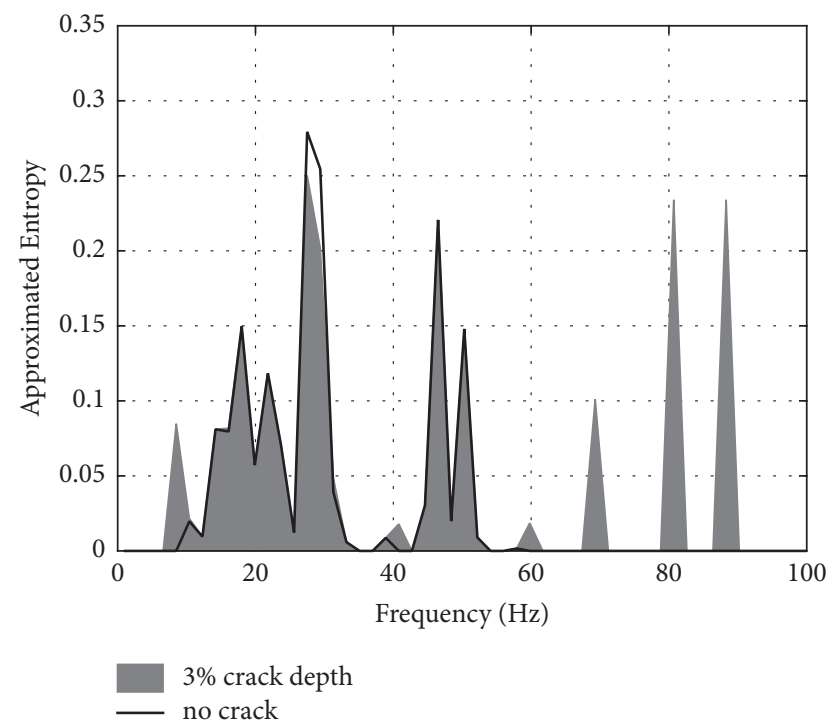

(b)

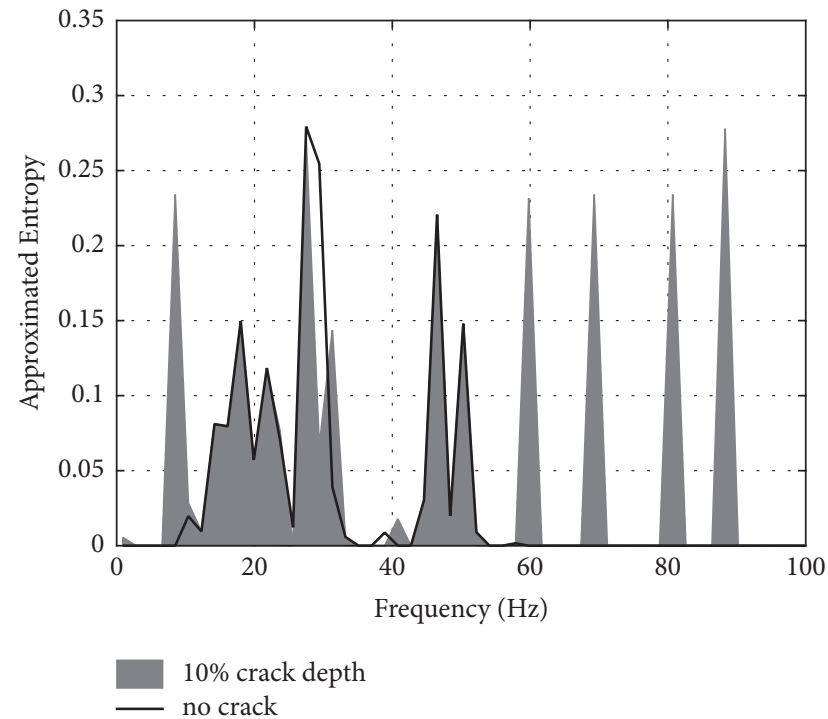

(d)

FIGURE 10: Approximated Entropy of the FFTs of the rotating system (SNR $\left.=1000, r=5 \times 10^{-5}\right)$. Comparison between the cases of healthy shaft and cracked shaft: (a) 1\% crack depth; (b) 3\% crack depth; (c) 5\% crack depth; (d) 10\% crack depth.

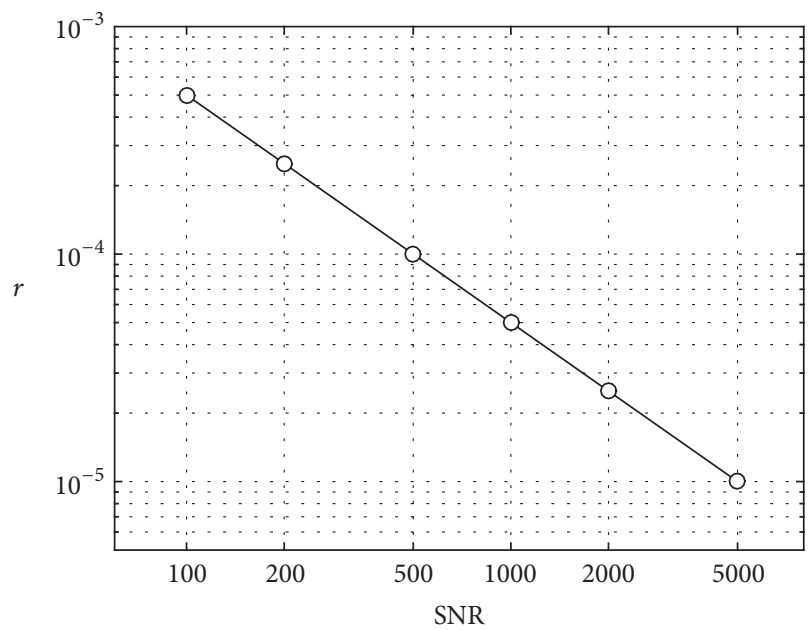

FIGURE 11: Values of the parameter $r$ that allows for crack detection for different levels of SNR in the FFTs. 


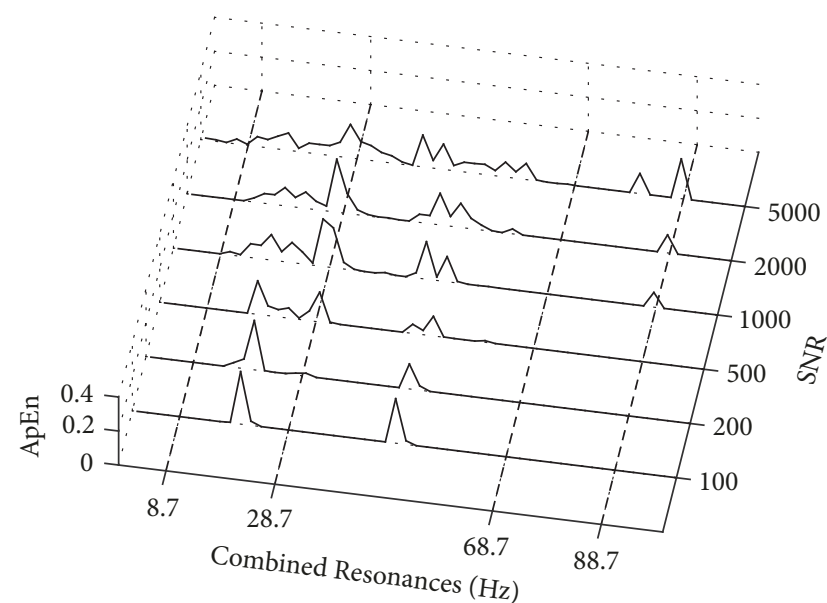

(a)

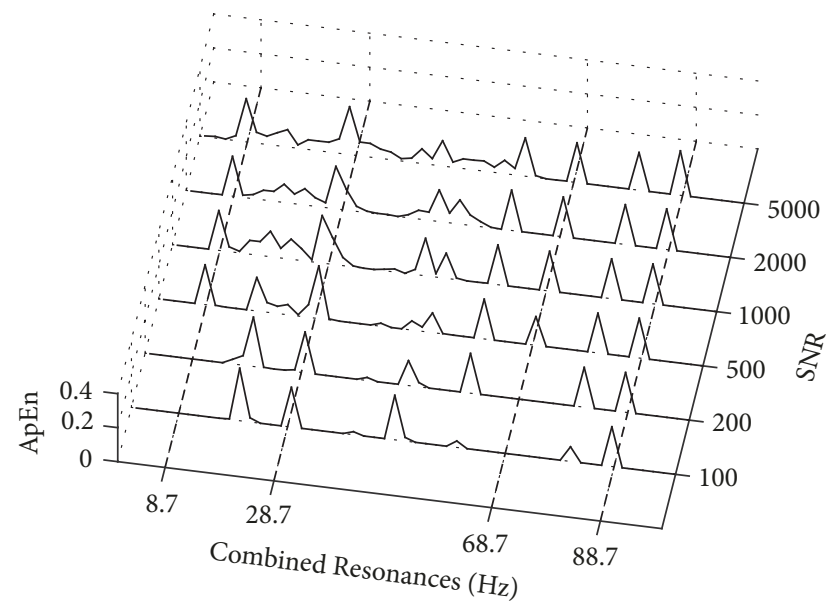

(c)

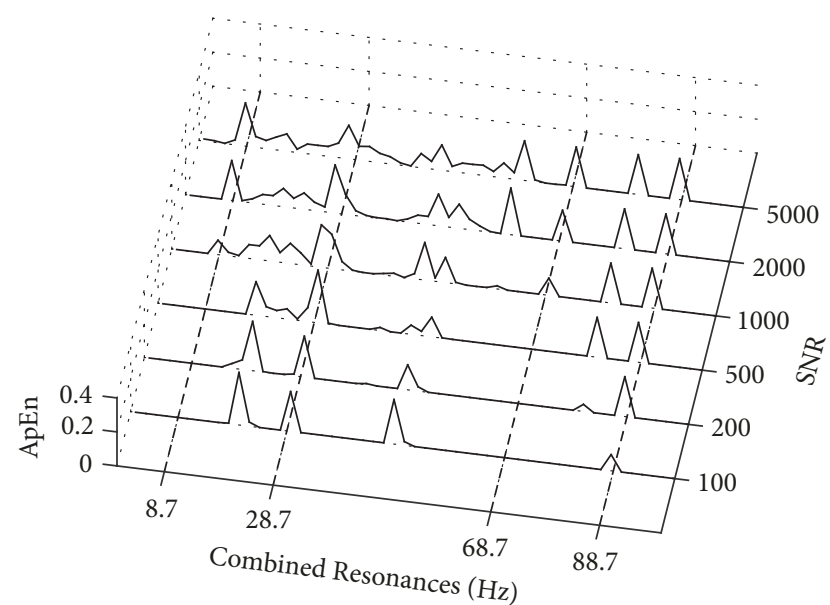

(b)

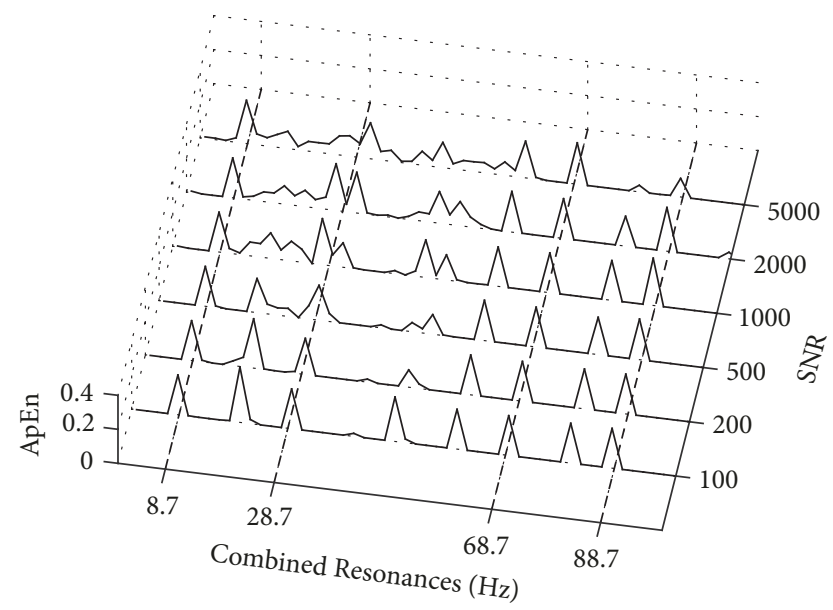

(d)

Figure 12: Approximated Entropy for different levels of SNR in the FFTs: (a) 1\% crack depth; (b) 3\% crack depth; (c) 5\% crack depth; (d) 10\% crack depth.

the peak at $88.7 \mathrm{~Hz}$ presents a significant variation as the process is repeated. The combination resonance at $88.7 \mathrm{~Hz}$ is not highlighted by the ApEn algorithm in many runs of the process, which is an indication that the detection of a crack at this stage can be jeopardized by the noise.

In the case of 3\% crack depth (Figure 13(b)), the peaks at $8.7 \mathrm{~Hz}$ and $68.7 \mathrm{~Hz}$ show significant variation, which is an evidence that they will not always appear in the ApEn curves. However, the peak at $88.7 \mathrm{~Hz}$ is always present (constant ApEn amplitude value). Therefore, the combination resonances can be detected as well as the presence of the crack. For the 5\% and 10\% crack depths (Figures 13(c) and 13(d)), all three peaks appear in the ApEn curves (amplitude values above 0.2 irrespective of the run). Hence, the detection of the crack is also possible. Therefore, the results in Figure 13 show that the methodology is robust enough for crack detection down to $3 \%$ crack depths in the shaft (regarding numerical simulations).

\section{Experimental Application}

The proposed crack detection technique was applied experimentally in the rotating machine of Figure 1(a). A small notch representing an open crack was machined in the shaft. The machining work resulted in a notch with, approximately, $0.5 \mathrm{~mm}$ thickness and $3.7 \mathrm{~mm}$ depth (22\% of the shaft diameter), as depicted in Figure 14. The notch was machined $50 \mathrm{~mm}$ from the disk located at node \#23. The crack was imposed at the position corresponding to the element between nodes \#19 and \#20 of the rotor FE model (see Figure 1(b)), as presented by Cavalini Jr. et al. [14]. Clearly, this notch is larger than cracks that arise in real rotors. However, many authors adopt the same procedure due to the difficulty of nucleating and propagating cracks for experimental purposes $[18,19]$.

Figure 15 shows the FFTs measured at the positions corresponding to nodes $\# 8$ and $\# 28$ of the FE model (horizontal vibration responses). The healthy (pristine condition) and cracked shaft conditions are presented, considering the 

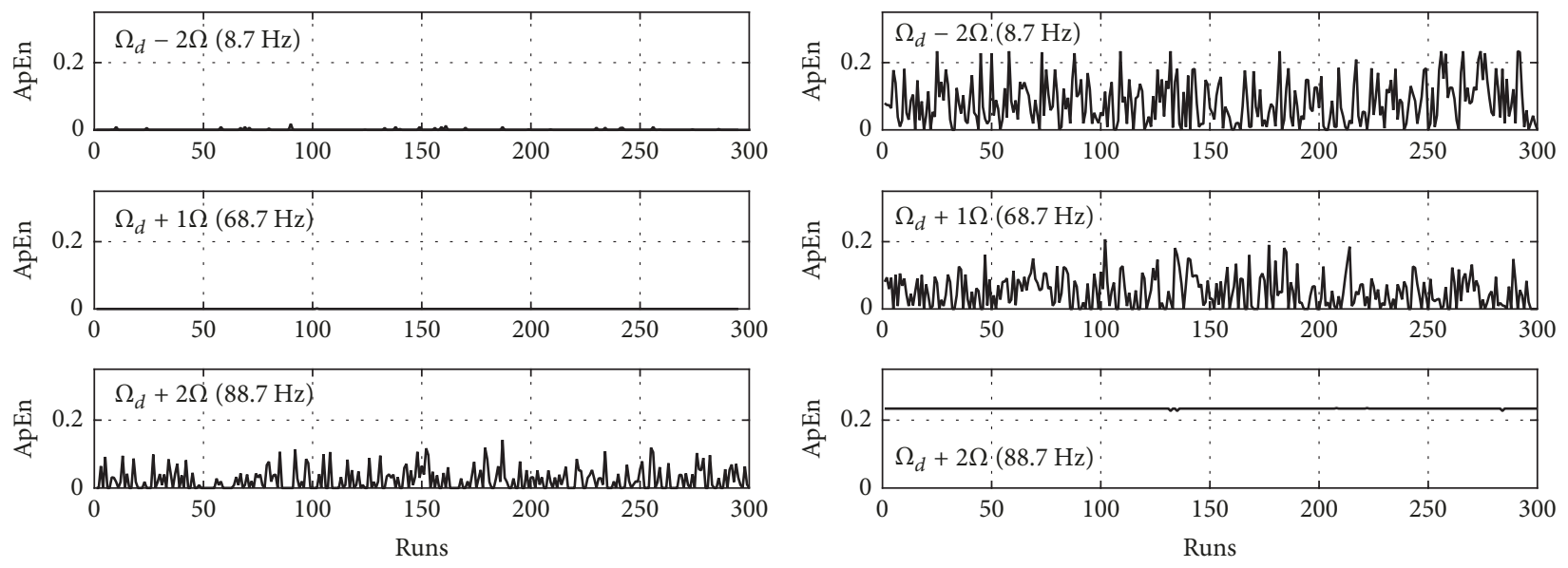

(a)
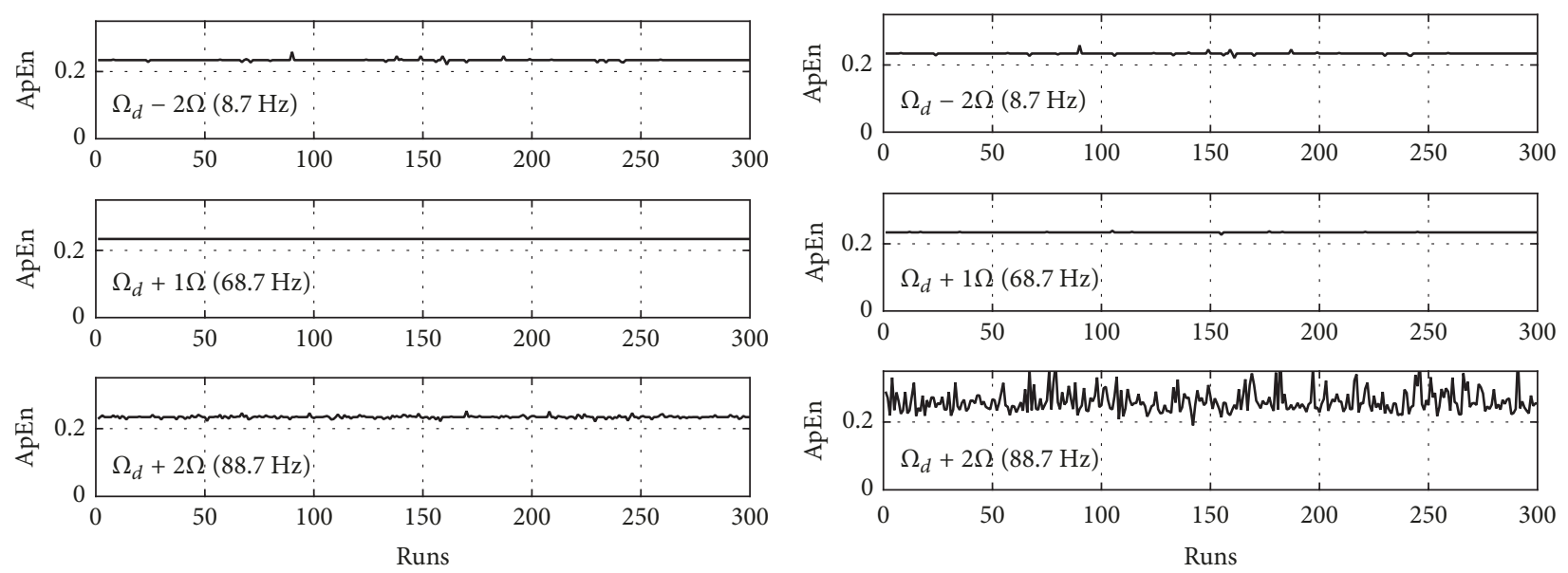

(c)

(d)

FiguRE 13: Approximated Entropy value of the combination resonances at $8.7 \mathrm{~Hz}, 68.7 \mathrm{~Hz}$, and $88.7 \mathrm{~Hz}\left(\mathrm{SNR}=1000, r=5 \times 10^{-5}\right):(\mathrm{a}) 1 \%$ crack depth; (b) $3 \%$ crack depth; (c) $5 \%$ crack depth; (d) $10 \%$ crack depth.

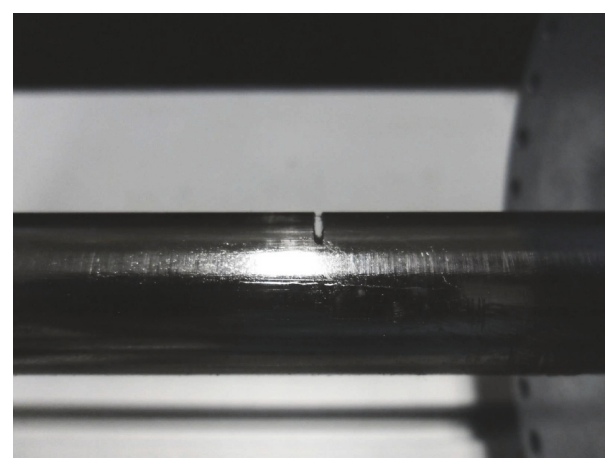

FIGURE 14: Small notch representing an open crack in the shaft of the test rig.

system subjected to the diagnostic harmonic force of $48.7 \mathrm{~Hz}$ frequency. In this case, the diagnostic force was applied in the horizontal direction at the bearing location with $25 \mathrm{~N}$ amplitude (similar to conditions adopted in the numerical analyses). The measurements were performed by the analyzer
Agilent ${ }^{\circledR}$ model $35670 \mathrm{~A}$ in the range of 0 to $200 \mathrm{~Hz}$ and frequency resolution of $0.25 \mathrm{~Hz}$.

The results of Figure 15 clearly show two main components in the spectrum, one being at $20 \mathrm{~Hz}$ related to the rotating speed $(1200 \mathrm{rpm})$ and another being at $48.7 \mathrm{~Hz}$ related to the diagnostic excitation. Note that peaks at frequencies of combination resonances can be seen even in the FFTs obtained from the healthy shaft, as well as superharmonics of the rotating speed $(2 \times, 3 \times$, and $4 \times$ vibration components). This behavior is associated with a $P$ type bearing used in the machine, which does not operate as a full self-alignment ball bearing. A similar result was presented by Ishida and Inoue [5] for conventional bearings. However, some differences in amplitude can be observed mainly at $-\Omega_{d}+3 \Omega(11.3 \mathrm{~Hz})$ and $\Omega_{d}+2 \Omega(88.7 \mathrm{~Hz})$ when the FFTs of the healthy and cracked shaft are compared.

Figure 16 presents the results obtained by applying the ApEn algorithm to the FFTs shown in Figure 15. In this case, $N=7$ (frequency at which the ApEn values are calculated), $p=2$ (size of the blocks in the ApEn algorithm), and $r=10^{-5}$ (tolerance adopted in the ApEn algorithm). 


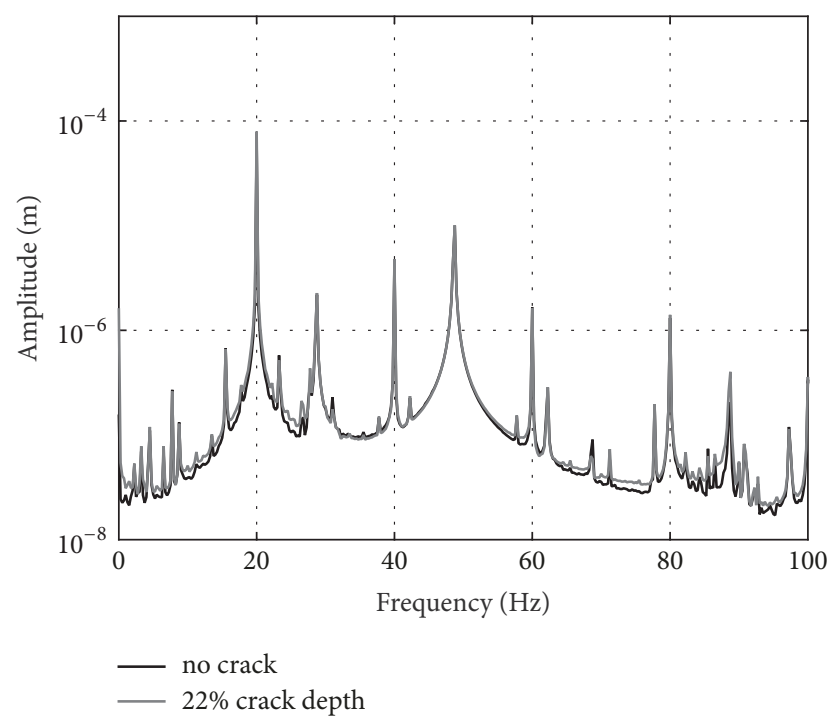

(a)

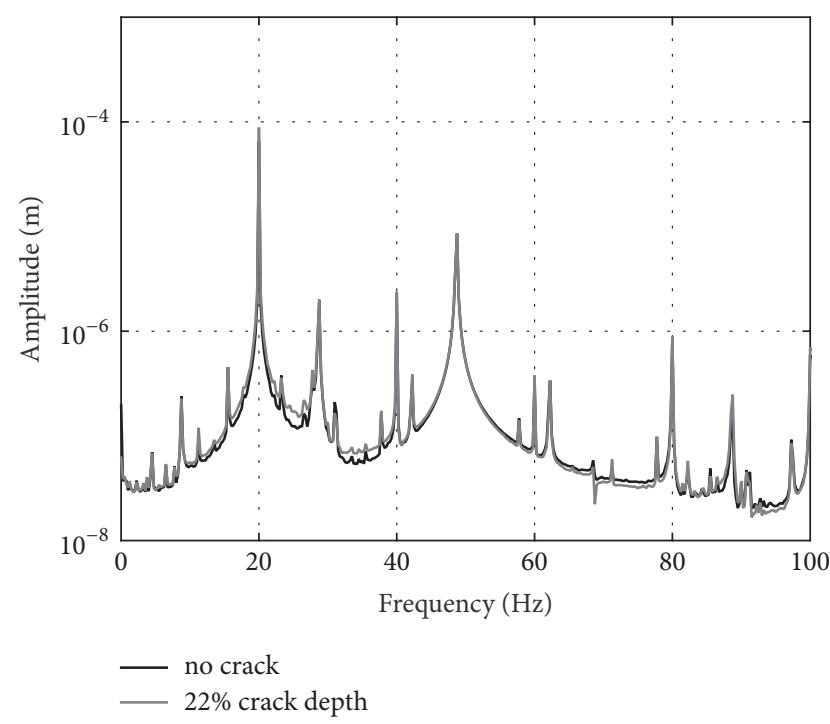

(b)

FIGURE 15: FFTs of the rotor vibration responses for the healthy and cracked shaft. Experimental responses as excited by the diagnostic frequency of $48.7 \mathrm{~Hz}$ at node \#4: (a) horizontal response at node \#8; (b) horizontal response at node \#28.

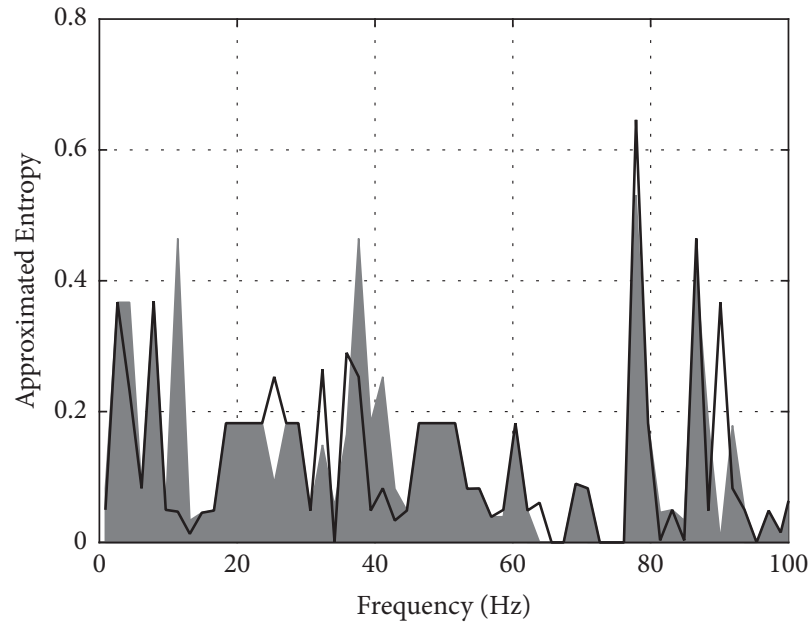

$22 \%$ crack depth no crack

(a)

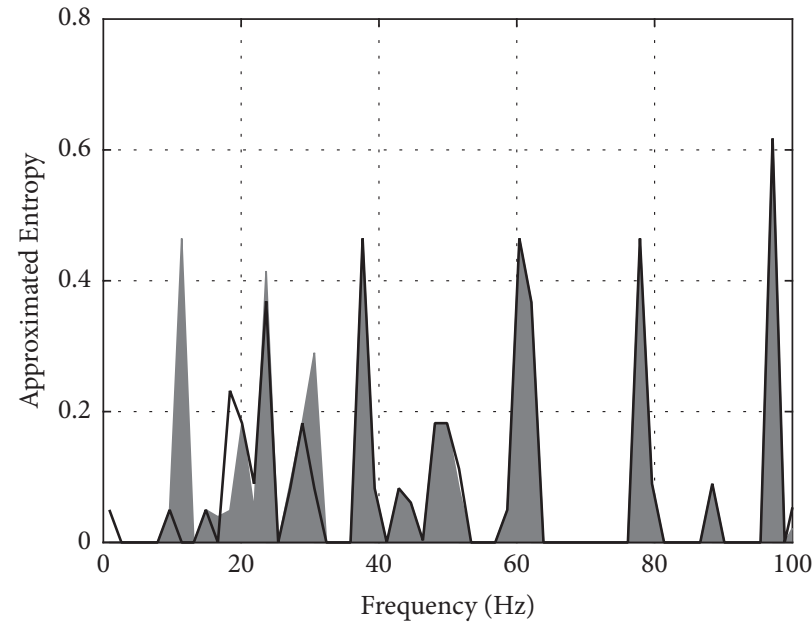

$22 \%$ crack depth no crack

(b)

Figure 16: Approximated Entropy of the experimental FFTs of the rotating system. Comparison between the cases of healthy and cracked shaft: (a) response at node $\# 8\left(N=7, p=2\right.$, and $\left.r=1 \times 10^{-8}\right)$; (b) response at node $\# 28\left(N=7, p=2\right.$, and $\left.r=5 \times 10^{-8}\right)$.

It is worth mentioning that the number of points used in the numerical applications was $N=19$ (ApEn values calculated every $2 \mathrm{~Hz}$ ). This value was reduced to $N=7$ in the experimental application (ApEn values calculated every $1.75 \mathrm{~Hz}$ ) to approximately keep the same frequency resolution used for the numerical results.

The results in Figure 16 show that the ApEn curves of the healthy shaft and the cracked shaft are very similar (given the high similarity of the FFTs - see Figure 15). However, the ApEn algorithm associated with the combination resonance approach was able to detect the existence of the crack experimentally. In both measurements at nodes \#8 and \#28, the presence of the combination resonance at $-\Omega_{d}+3 \Omega$ $(11.3 \mathrm{~Hz})$ is clear. At node \#8 (Figure 16(a)), an additional variation of the ApEn value can be observed around the first natural frequency of the shaft $(28.7 \mathrm{~Hz})$ and in the second harmonics of rotation $(40 \mathrm{~Hz})$. These frequencies are not associated with any combination resonances, but their amplitudes also changed by the existence of the crack.

\section{Conclusion}

In this paper, a crack detection technique devoted to rotating machines was proposed. The combination resonances 
approach was associated with the ApEn algorithm, resulting in a methodology suitable for industrial applications. Differently from the ApEn technique previously presented in literature, where the rotor should run in nonstationary conditions (run-up or run-down), here the rotor can operate at nominal rotating speeds during the tests.

Numerical simulations were performed by considering incipient cracks $(1 \%, 3 \%, 5 \%$, and $10 \%$ crack depths). The results showed that the algorithm is able to detect the existence of the cracks by highlighting the combination resonances down to $3 \%$ crack depths, even in the presence of noise in the FFTs. In this case, the tolerance parameter $r$ must be chosen according to the level of noise for achieving good results.

In the experimental tests, the algorithm was also able to highlight the combination of resonances and, therefore, the existence of the crack in the shaft. Despite the high similarity between the FFTs of the healthy shaft and the cracked shaft, the algorithm allowed the detection of a crack of $22 \%$ crack depth in the shaft.

Besides the satisfactory results obtained in the presence of noise (see the numerical analyses for $1 \%$ to $5 \%$ crack depths), the detection of incipient cracks can be challenging in industrial environments. It is worth mentioning that the influence of the crack should be present in the vibration responses that are measured in the rotating machine. Consequently, further investigations are necessary to determine the crack detection threshold associated with the proposed methodology.

It is expected that satisfactory results are obtained by using the conveyed approach even in rotating systems with gearboxes, in which the measured spectral responses typically present a high density of information. As discussed along the paper, the proposed technique is based on comparisons between the actual condition of the machine and a baseline. Thus, variations on the measured spectral responses and, consequently, on the determined Approximated Entropy are investigated.

Finally, the obtained results demonstrated that the proposed technique can detect cracks in rotating shafts. However, further research effort will be dedicated to a more comprehensive experimental validation of the proposed methodology (e.g., by adopting smaller cracks in the shaft).

\section{Nomenclature}

$\begin{array}{ll}\text { ApEn: } & \text { Approximated Entropy } \\ \mathbf{D}: & \text { Damping matrix } \\ \mathbf{D}_{g}: & \text { Gyroscopic matrix } \\ \mathbf{f}_{e}: & \text { Vector of excitation forces } \\ \mathbf{f}_{u}: & \text { Vector of unbalance forces } \\ \mathbf{f}_{w}: & \text { Vector of weight forces } \\ \mathbf{K}: & \text { Stiffness matrix } \\ \mathbf{K}_{\mathrm{CR}}: & \text { Crack flexibility matrix } \\ \mathbf{M}: & \text { Inertia matrix } \\ n: & \text { Multiple of the rotating speed } \\ N: & \text { Number of points of the data series for } \\ & \text { ApEn calculation } \\ p: & \text { Number of points in each block for ApEn } \\ & \text { calculation }\end{array}$

q: Vector of generalized coordinates

$r$ : Tolerance in the ApEn algorithm

RMS: Root mean square of the signal

SNR: Signal-to-noise ratio

$\omega_{\Omega}: \quad$ Critical speed $\left[\mathrm{rad} \cdot \mathrm{s}^{-1}\right]$

$\Omega: \quad$ Rotating speed $\left[\mathrm{rad} \cdot \mathrm{s}^{-1}\right]$

$\Omega_{d}: \quad$ Excitation frequency $\left[\mathrm{rad} \cdot \mathrm{s}^{-1}\right]$.

\section{Data Availability}

The data used to support the findings of this study are available from the corresponding author upon request.

\section{Conflicts of Interest}

The authors declare that there are no conflicts of interest regarding the publication of this paper.

\section{Acknowledgments}

This project was supported by the Brazilian research foundations Conselho Nacional de Desenvolvimento Científico e Tecnológico (CNPq/304546-2017-8), Coordenação de Aperfeiçoamento de Pessoal de Nível Superior (CAPES), and Fundação de Amparo a Pesquisa do Estado de Minas Gerais (FAPEMIG/TEC-APQ-02284-15/TEC-APQ-00464-16), through the INCT-EIE.

\section{References}

[1] D. E. Bently and C. T. Hatch, Fundamentals of rotating machinery diagnostics, Bently Pressurized Bearing Company, Bently Pressurized Bearing Company, Minden, NV, USA, 2002.

[2] N. Bachschmid, P. Pennacchi, and E. Tanzi, Cracked Rotors: A Survey on Static and Dynamic Behaviour Including Modelling and Diagnosis, Springer-Verlag, Berlin, Germany, 2010.

[3] A. S. Sekhar, "Crack identification in a rotor system: a modelbased approach," Journal of Sound and Vibration, vol. 270, no. 4-5, pp. 887-902, 2004.

[4] J.-J. Sinou, "Detection of cracks in rotor based on the $2 \times$ and $3 \times$ super-harmonic frequency components and the crackunbalance interactions," Communications in Nonlinear Science and Numerical Simulation, vol. 13, no. 9, pp. 2024-2040, 2008.

[5] Y. Ishida and T. Inoue, "Detection of a rotor crack using a harmonic excitation and nonlinear vibration analysis," Journal of Vibration and Acoustics, vol. 128, no. 6, pp. 741-749, 2006.

[6] W. Lu and F. Chu, "Shaft crack identification based on vibration and AE signals," Shock and Vibration, vol. 18, no. 1-2, pp. 115-126, 2011.

[7] S. Seibold and K. Weinert, "A time domain method for the localization of cracks in rotors," Journal of Sound and Vibration, vol. 195, no. 1, pp. 57-73, 1996.

[8] A. S. Sekhar, "Detection and monitoring of crack in a coastdown rotor supported on fluid film bearings," Tribology International, vol. 37, no. 3, pp. 279-287, 2004.

[9] J. K. Sinha, "Higher order spectra for crack and misalignment identification in the shaft of a rotating machine," Structural Health and Monitoring, vol. 6, no. 4, pp. 325-334, 2007.

[10] Z. Kulesza and J. T. Sawicki, "Auxiliary state variables for rotor crack detection," Journal of Vibration and Control, vol. 17, no. 6, pp. 857-872, 2011. 
[11] G. Mani, D. D. Quinn, and M. Kasarda, "Active health monitoring in a rotating cracked shaft using active magnetic bearings as force actuators," Journal of Sound and Vibration, vol. 294, no. 3, pp. 454-465, 2006.

[12] J. T. Sawicki, M. I. Friswell, Z. Kulesza, A. Wroblewski, and J. D. Lekki, "Detecting cracked rotors using auxiliary harmonic excitation," Journal of Sound and Vibration, vol. 330, no. 7, pp. 1365-1381, 2011.

[13] J. T. Sawicki, D. L. Storozhev, and J. D. Lekki, "Exploration of NDE properties of AMB supported rotors for structural damage detection," Journal of Engineering for Gas Turbines and Power, vol. 133, no. 10, pp. 1-9, 2011.

[14] A. A. Cavalini Jr., L. Sanches, N. Bachschmid, and V. Steffen Jr., "Crack identification for rotating machines based on a nonlinear approach," Mechanical Systems and Signal Processing, vol. 79, pp. 72-85, 2016.

[15] D. L. Sampaio and R. Nicoletti, "Detection of cracks in shafts with the Approximated Entropy algorithm," Mechanical Systems and Signal Processing, vol. 72-73, pp. 286-302, 2016.

[16] I. W. Mayes and W. G. R. Davies, "Analysis of the response of a multi-rotor-bearing system containing a transverse crack in a rotor," Journal of Vibration, Acoustics, Stress, and Reliability in Design, vol. 106, no. 1, pp. 139-145, 1984.

[17] S. M. Pincus, "Approximate entropy as a measure of system complexity," Proceedings of the National Acadamy of Sciences of the United States of America, vol. 88, no. 6, pp. 2297-2301, 1991.

[18] P. N. Saavedra and L. A. Cuitio, "Vibration analysis of rotor crack identification," Journal of Vibration and Control, vol. 8, pp. 51-57, 2002.

[19] C. A. Papadopoulos, "Some comments on the calculation of the local flexibility of cracked shafts," Journal of Sound and Vibration, vol. 278, no. 4-5, pp. 1205-1211, 2004. 


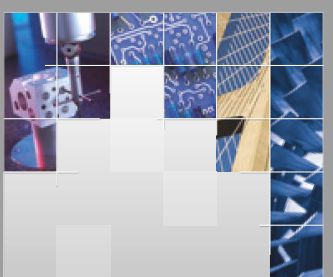

\section{Enfincering}
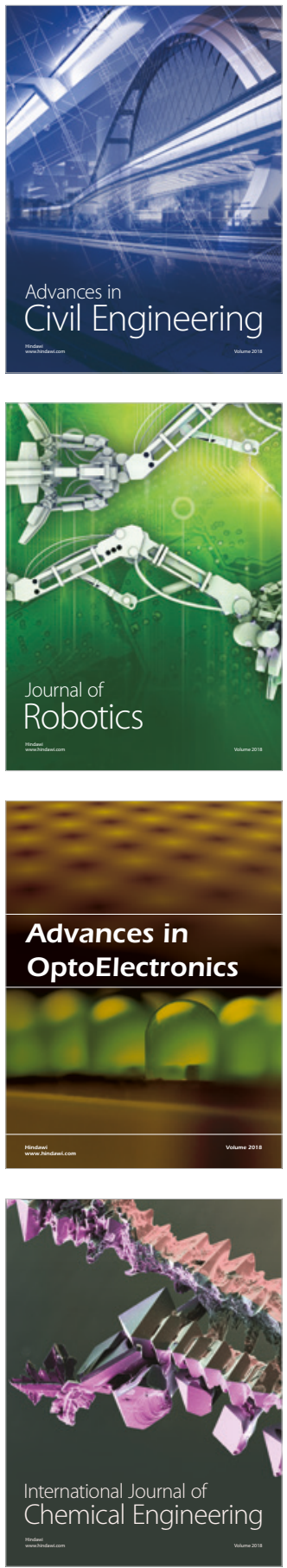

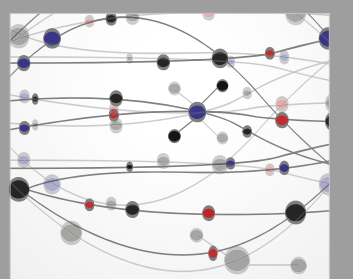

\section{Rotating \\ Machinery}

The Scientific World Journal

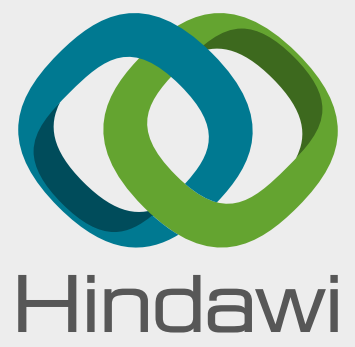

Submit your manuscripts at

www.hindawi.com
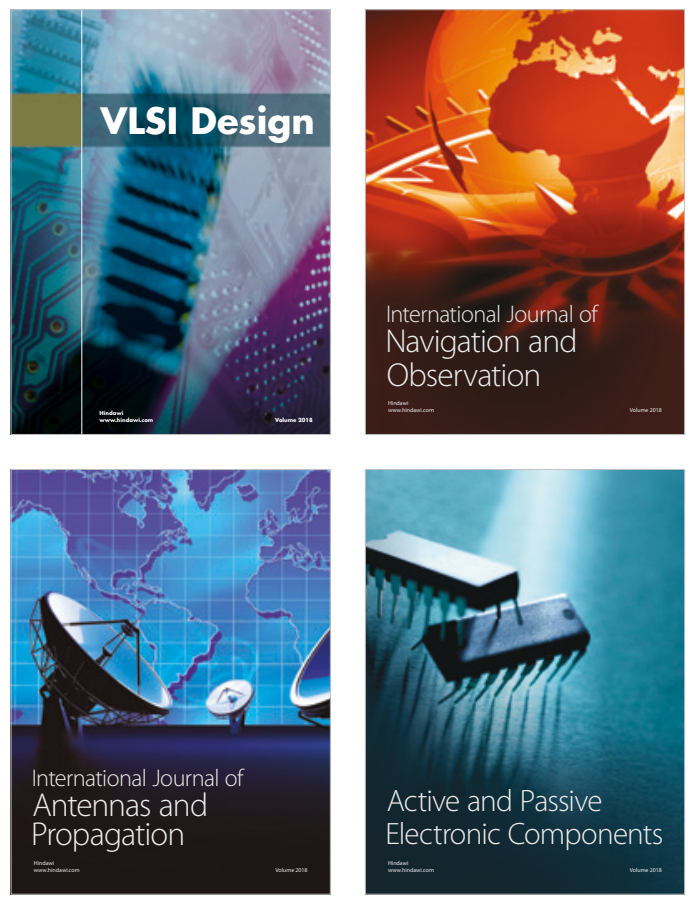
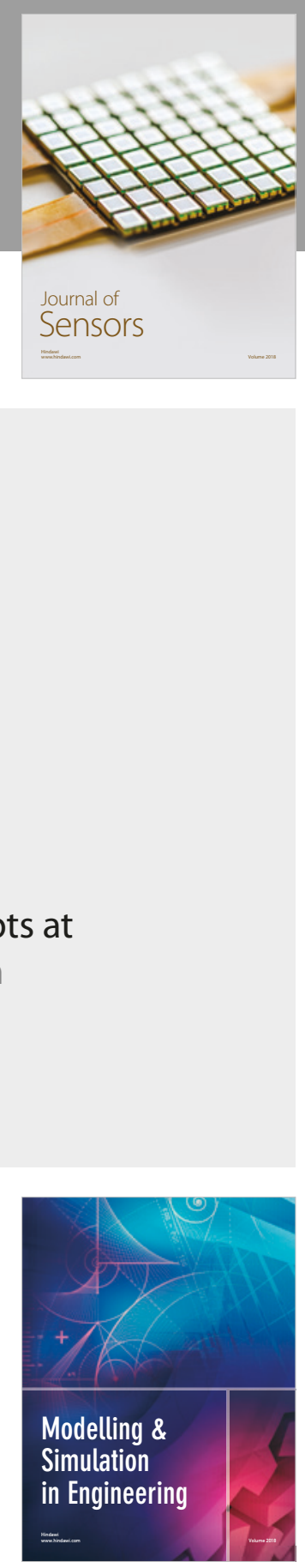

\section{Advances \\ Multimedia}
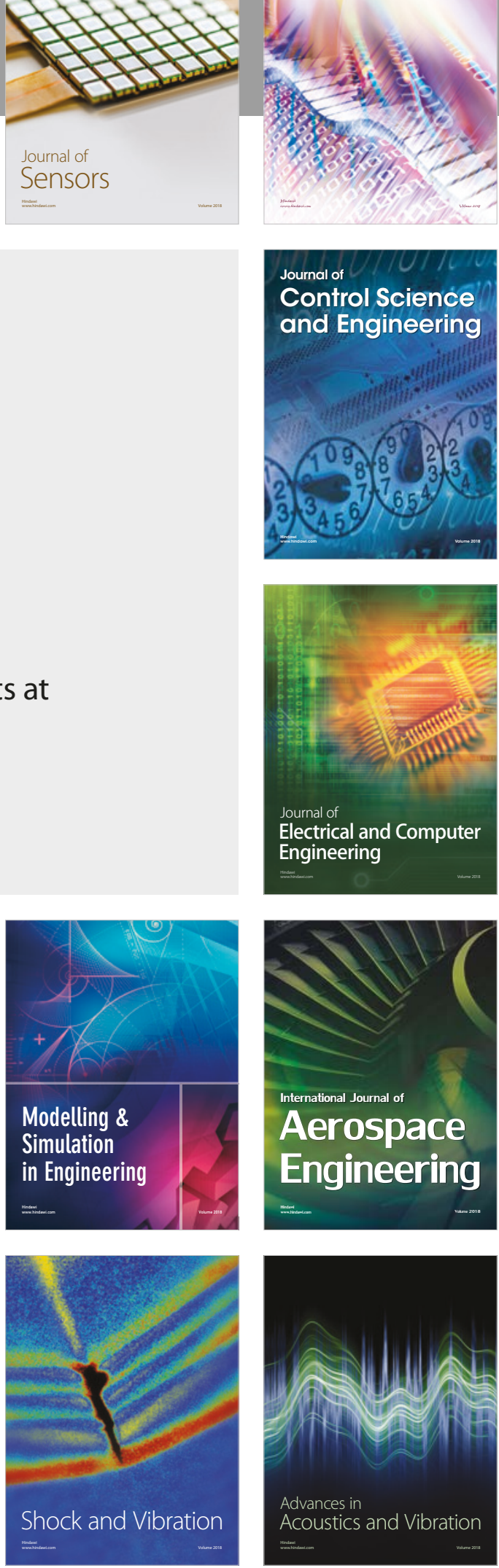